\title{
Stiff stroma increases breast cancer risk by inducing the oncogene ZNF217
}

\author{
Jason J. Northey, ${ }^{1,2}$ Alexander S. Barrett, ${ }^{3}$ Irene Acerbi, ${ }^{1,2}$ Mary-Kate Hayward, ${ }^{1,2}$ Stephanie Talamantes, ${ }^{1,2}$ Ivory S. Dean, ${ }^{1,2}$ \\ Janna K. Mouw, ${ }^{1,2}$ Suzanne M. Ponik, ${ }^{4}$ Jonathon N. Lakins, ${ }^{1,2}$ Po-Jui Huang, ${ }^{1,2}$ Junmin Wu, ${ }^{5}$ Quanming Shi, ${ }^{6}$ Susan Samson, ${ }^{7}$ \\ Patricia J. Keely, ${ }^{4}$ Rita A. Mukhtar, ${ }^{1}$ Jan T. Liphardt, ${ }^{6}$ John A. Shepherd, ${ }^{8}$ E. Shelley Hwang, ${ }^{9}$ Yunn-Yi Chen, ${ }^{10}$ Kirk C. Hansen, ${ }^{3,11}$ \\ Laurie E. Littlepage, ${ }^{5}$ and Valerie M. Weaver ${ }^{1,2,7,8,12}$
}

\begin{abstract}
'Department of Surgery, ${ }^{2}$ Center for Bioengineering and Tissue Regeneration, UCSF, San Francisco, California, USA. ${ }^{3}$ Department of Biochemistry and Molecular Cenetics, University of Colorado Denver, Anschutz Medical Campus, Aurora, Colorado, USA. ${ }^{4}$ Department of Cell and Regenerative Biology, University of Wisconsin-Madison, Madison, Wisconsin, USA. ${ }^{5}$ Harper Cancer Research Institute, Department of Chemistry and Biochemistry, University of Notre Dame, South Bend, Indiana, USA. ${ }^{6}$ Department of Bioengineering, Stanford University, Palo Alto, California, USA. ${ }^{7}$ Helen Diller Comprehensive Cancer Center, UCSF, San Francisco, California, USA. ${ }^{8}$ Population Sciences in the Pacific Program (Cancer Epidemiology), University of Hawaii Cancer Center, University of Hawaii at Manoa, Manoa, Hawaii, USA. ${ }^{9}$ Department of Surgery, Duke University Medical Center, Durham, North Carolina, USA. ${ }^{10}$ Department of Pathology, UCSF, San Francisco, California, USA. "Division of Medical Oncology, Department of Medicine, University of Colorado Anschutz Medical Campus, Aurora, Colorado, USA. ${ }^{12}$ Radiation Oncology, Eli and Edythe Broad Center of Regeneration Medicine and Stem Cell Research, UCSF, San Francisco, California, USA.
\end{abstract}

\begin{abstract}
Women with dense breasts have an increased lifetime risk of malignancy that has been attributed to a higher epithelial density. Quantitative proteomics, collagen analysis, and mechanical measurements in normal tissue revealed that stroma in the high-density breast contains more oriented, fibrillar collagen that is stiffer and correlates with higher epithelial cell density. microRNA (miR) profiling of breast tissue identified miR-203 as a matrix stiffness-repressed transcript that is downregulated by collagen density and reduced in the breast epithelium of women with high mammographic density. Culture studies demonstrated that ZNF217 mediates a matrix stiffness- and collagen density-induced increase in Akt activity and mammary epithelial cell proliferation. Manipulation of the epithelium in a mouse model of mammographic density supported a causal relationship between stromal stiffness, reduced miR-203, higher levels of the murine homolog Zfp217, and increased Akt activity and mammary epithelial proliferation. ZNF217 was also increased in the normal breast epithelium of women with high mammographic density, correlated positively with epithelial proliferation and density, and inversely with miR-203. The findings identify ZNF217 as a potential target toward which preexisting therapies, such as the Akt inhibitor triciribine, could be used as a chemopreventive agent to reduce cancer risk in women with high mammographic density.
\end{abstract}

\section{Introduction}

High mammographic density (MD) associates with elevated lifetime risk of malignancy (1-4). Every $3 \%$ to $6 \%$ increase in MD corresponds to a $10 \%$ increase in relative risk for breast cancer $(5,6)$. Nevertheless, the molecular mechanisms whereby MD promotes breast cancer risk remain unclear. High breast density correlates with an increase in glandular epithelial content (7-13). The presence of more epithelial cells representing units of oncogenesis is proportional to the enhanced risk documented for women with high MD $(14,15)$. Estrogenic hormones regulate epithelial proliferation and systemically elevated estrogen is causally linked to increased breast cancer incidence $(16,17)$. Consistently, tamoxifen treatment, which interferes with estrogen function, decreases breast cancer risk while concomitantly reducing MD and epithelial density $(18,19)$. Although studies have shown that the proportional risk reduction for invasive breast cancer is almost $50 \%$ with 5 years of tamoxifen or raloxifene use, the use of these agents for

Conflict of interest: The authors have declared that no conflict of interest exists. Copyright: ( 2020, American Society for Clinical Investigation.

Submitted: April 2, 2019; Accepted: July 14, 2020; Published: September 28, 2020.

Reference information: J Clin Invest. 2020;130(11):5721-5737.

https://doi.org/10.1172/JCl129249. chemoprevention is less than $0.1 \%(20,21)$. This minimal uptake of potentially effective chemoprevention can be directly attributed to poor risk stratification tools that do not allow for identification of those patients likely to benefit most from chemoprevention. Better discrimination of $\mathrm{MD}$-associated risk could also reduce unnecessary tamoxifen treatment and its associated potential for long-term toxicity. Nevertheless, breast cancer risk conferred by MD exceeds that of all other known risk factors aside from age and genetic mutation. Paradoxically, while a large fraction of breast cancer patients also have dense breasts, so do many women (30\%) who do not get cancer $(4,22)$. The ability to discriminate between these populations based on an understanding of the biological mediators that comprise the risk associated with MD would meet an imperative clinical need to stratify breast cancer risk and would in the future allow improved decision making for patients contemplating risk reduction strategies.

MD is a heritable trait. Approximately $60 \%$ of the MD phenotype of breast tissue can be attributed to systemic or tissuespecific intrinsic genetic factors (23-26). One inherited phenotype associated with MD is a dense interstitial stroma characterized by abundant type I interstitial fibrillar collagen (9, 13, 27-29). Interstitial collagen contributes to the mechanical integrity of the tissue and higher levels of fibrillar and cross-linked collagen increase the 
A
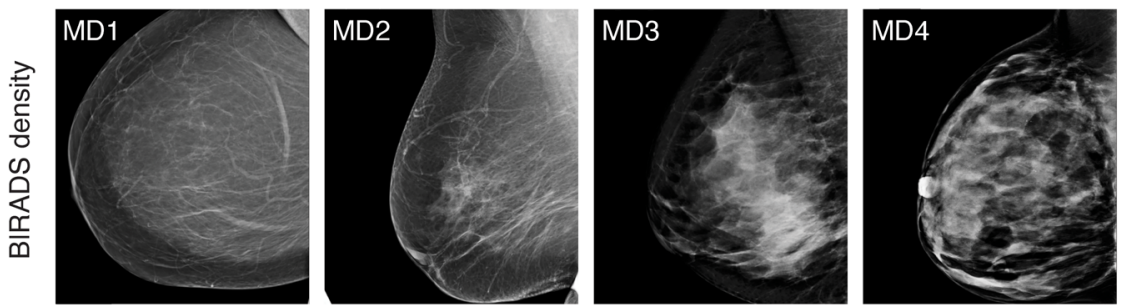

Mammographic density and breast cancer risk

B

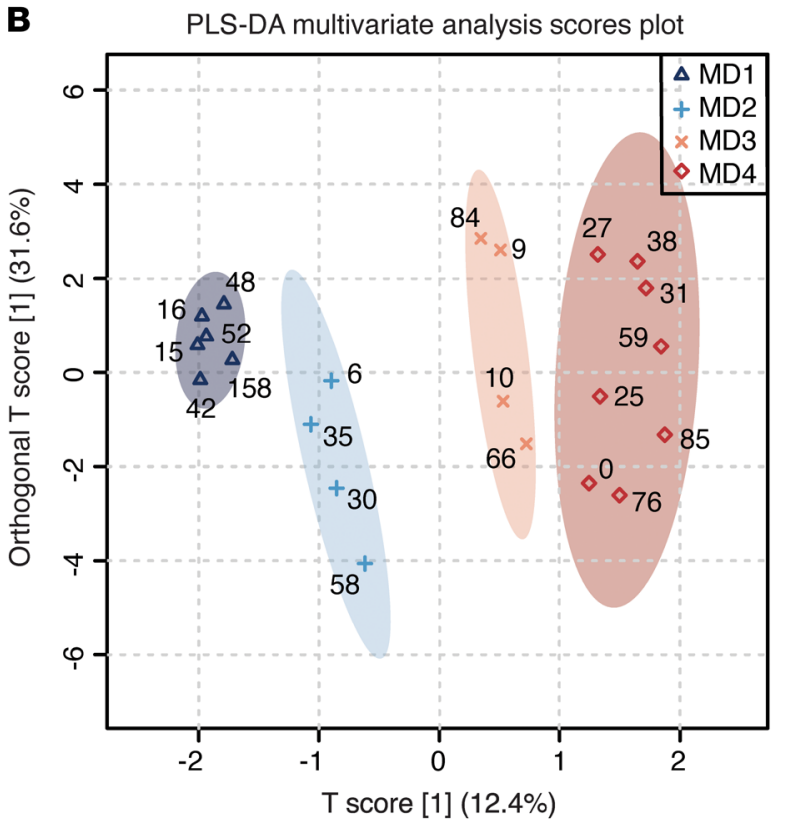

D

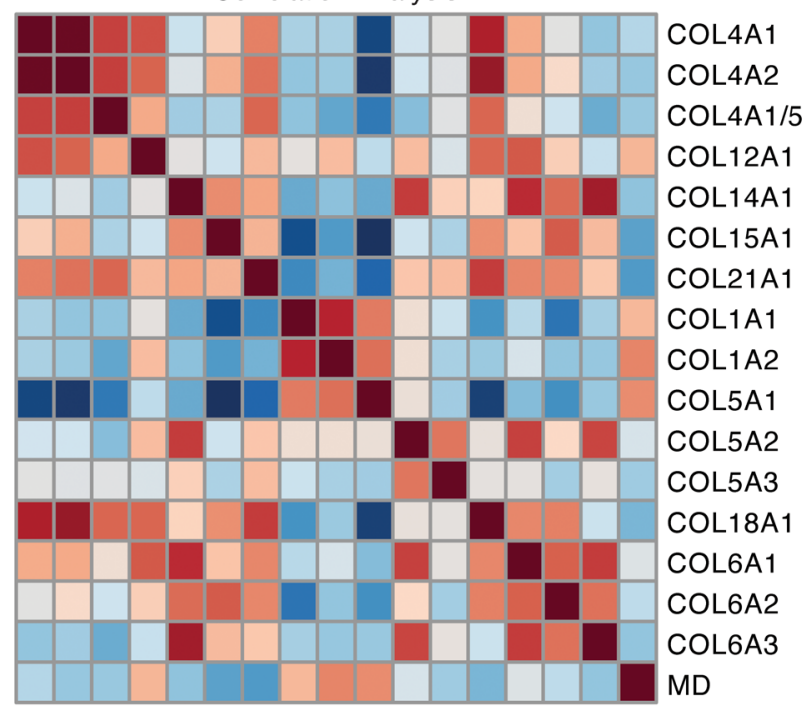

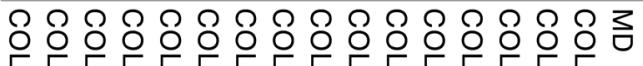

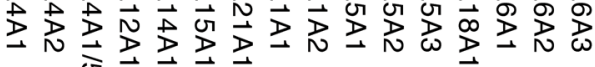

C Variable of importance (VIP) plots from PLS-DA analysis
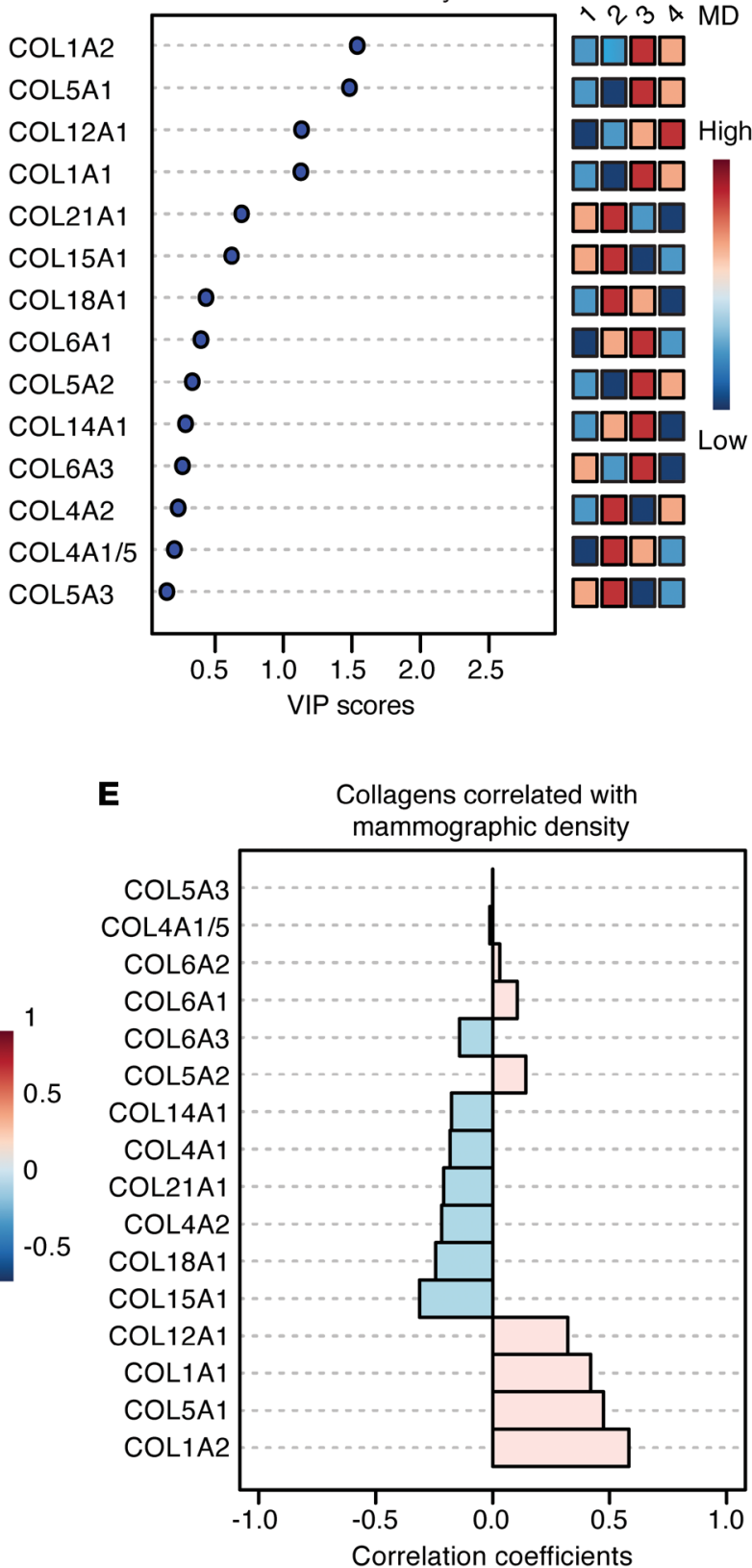
Figure 1. Quantitative proteomics reveal that breast density correlates with more abundant fibrillar collagens. (A) Mammography showing the 4 categories of mammographic density (MD) as measured using the Breast Imaging Reporting and Data System (BIRADS). (B) A multivariate analysis (partial least squares-discriminant analysis, PLS-DA) of proteomic data using the indicated prophylactic mastectomy tissues (MD1, $n=6$; MD2, $n=4$; MD3, $n=4$; MD4, $n=8$ for all panels). (C) Variable of importance (VIP) plots were generated to rank the collagens most associated with high MD. (D) Analysis examining the correlation between MD and the levels of different collagens presented as a heatmap and accompanied by a graph of correlation coefficients (E). Dark blue to dark red shades represent weaker to stronger correlations.

stiffness of the tissue stroma (30-32). Computational analysis of a large cohort of mammographic images suggested that the collagen-rich breast tissue of women with high MD is stiffer than that of the women with low MD (28). Invasive ductal carcinoma (IDC) is characterized by higher amounts of remodeled and stiffened collagen that increases with tumor aggression (30). Experimental models causally implicate a stiffened extracellular matrix (ECM) in malignant transformation and metastasis through mechanosignaling-mediated enhancement of cell growth, survival, and migration (31, 33-35). A stiff ECM can also induce molecular and epigenetic changes in cells that include altering levels of microRNAs (miRs) and tumor suppressors and stimulating developmental programs that lead to sustained phenotypic changes associated with tumor aggression, including a mesenchymal-like transition $(33,36-39)$. Thus, a stiffer breast tissue stroma could increase risk of malignancy by increasing epithelial density and reprogramming the breast epithelium toward a preoncogenic high-risk state.

We analyzed the biochemical and biophysical properties of the ECM in normal, primarily healthy human breast tissue representing the range of MDs and linked these measurements to biological and molecular features in the stroma and epithelium. Causal associations between ECM stiffness and epithelial genotype and phenotype were established using mammary organoids and a mouse model that recapitulates features of a high-density collagen-rich breast stroma. The studies further identified a tension-mediated mechanism that fosters mammary epithelial cell (MEC) growth and predisposes the epithelium to transformation by regulating levels of an oncogene implicated in breast tumor aggression and for which an antitumor treatment already exists. The work has led us to conclude that the mechanically primed high-MD breast stroma can alter both the nature and the abundance of the breast epithelium. We contend that understanding the contribution of tissue tension to breast cancer risk constitutes an alternative paradigm that could allow for the identification of additional biomarkers of risk, and importantly, the development and/or application of novel chemoprevention targets.

\section{Results}

The high-MD breast stroma is stiffer and contains more linearized and bundled fibrillar collagen. To assess the relationship between $\mathrm{MD}$, the ECM, and breast cancer risk, we analyzed the ECM protein composition, organization, and mechanical properties from normal female breast tissue. Histologically normal breast tissue $(n=22)$ was collected from premenopausal women undergoing prophylactic mastectomy $(n=16)$ and contralateral prophylactic mastectomy $(n=7)$ (Supplemental Table 1; supplemental material available online with this article; https://doi.org/10.1172/ JCI129249DS1). These tissue samples were selected to represent the spectrum of the Breast Imaging-Reporting and Data System (BIRADS) used to classify MD. Prior to surgery, the tissues were classified as MD1 (almost entirely fatty; $n=6$ ), MD2 (scattered density; $n=4$ ), MD3 (heterogeneously dense; $n=4$ ), and MD4 (extremely dense; $n=8$; Figure 1A). Pathological examination confirmed that the majority of these breast tissues were disease-free (no atypia, dysplasia, premalignancy, invasive carcinoma, fibrocystic disease, or infection [68.3\%]; with 3/22 from women with verified BRCA1 germline mutations [13.6\%]). Two of the women had either adjacent adenocarcinoma or IDC (9\%), and 5 of the women had IDC or ductal carcinoma in situ (DCIS) in the contralateral breast $(22.7 \%)$, conditions that could present an abnormal baseline breast tissue phenotype.

To analyze the ECM proteins differentially expressed in these tissues, the samples were subjected to mass spectrometry-based proteomic analysis to profile the insoluble ECM and the chaotrope-soluble matrisome of ECM-associated proteins. Multivariate evaluation of the resultant proteomics data using partial least squares-discriminant analysis (PLS-DA) distinguished the tissue classified within each of the 4 BIRADS MD categories according to their ECM quantity and composition (Figure 1B and Supplemental Table 2). Visualization of the variable of importance (VIP) was used to highlight the extracellular collagens identified by the PLS-DA analysis. Several fibrillar collagens (COL1A2, COL1A1, COL5A1) and COL12A1, a fibril-associated collagen with interrupted triple helices (FACIT), were abundantly expressed in tissues from the highest MD group (MD4; Figure 1C). Correlation analysis of the proteomics data revealed that the structural collagens (COL1A2, COL1A1, COL5A1, and COL12A1) and injury-associated collagens (COL5A2, COL6A1, and COL6A2) were the ECM proteins that correlated the most significantly with the high-MD classification (Figure 1, D and E). Consistent with an injury-primed ECM, the high-MD breast tissue also contained more of the glycoproteins ECM1, MGP, and dermatopontin (DPT), as well as the proteoglycans lumican (LUM) and biglycan (BGN) (Supplemental Figure 1).

Noticeably, ECM quantity and quality strongly distinguished the tissue classified as MD1 from the MD4 tissue. The biospecimens classified as MD3 clustered more closely with MD4, and the MD2 group clustered more closely with the MD1 group, echoing prior studies that grouped MD1 and MD2 specimens as low MD and MD3 and MD4 as high MD.

Further analysis of the collagen ECM supported differences based on MD. Trichrome staining confirmed that the breast stroma classified as MD3 and MD4 (high MD) contained more total stromal collagen than either of the breast tissue specimens classified as MD1 and MD2 (low MD) (Figure 2, A and B). The levels of the fibrillar collagens COL1A1, COL1A2, and COL5A1 were also greater in the high-MD tissue than in the low-MD breast tissue (Figure 2, C-E). Quantification of polarized images of picrosirius red-stained tissue revealed that the high-MD tissue contained more fibrillar collagen than the low-MD tissue (Figure 2, F and G). Second harmonic generation (SHG) imaging of tissues using 2-photon microscopy and Imaris image analysis further revealed 
A
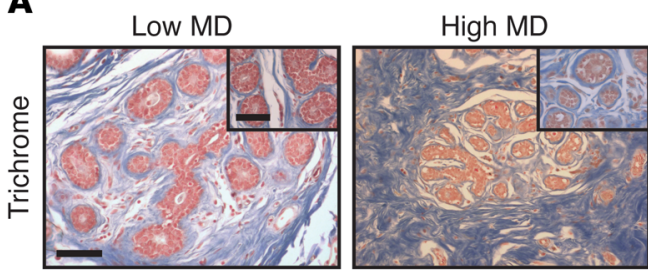

B

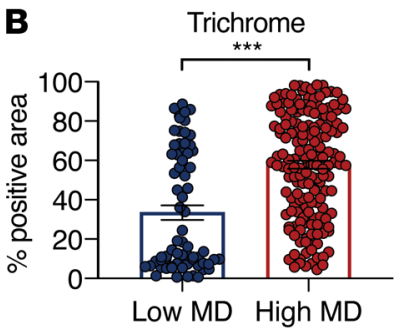

Fibrillar collagens

C COL1A1

D
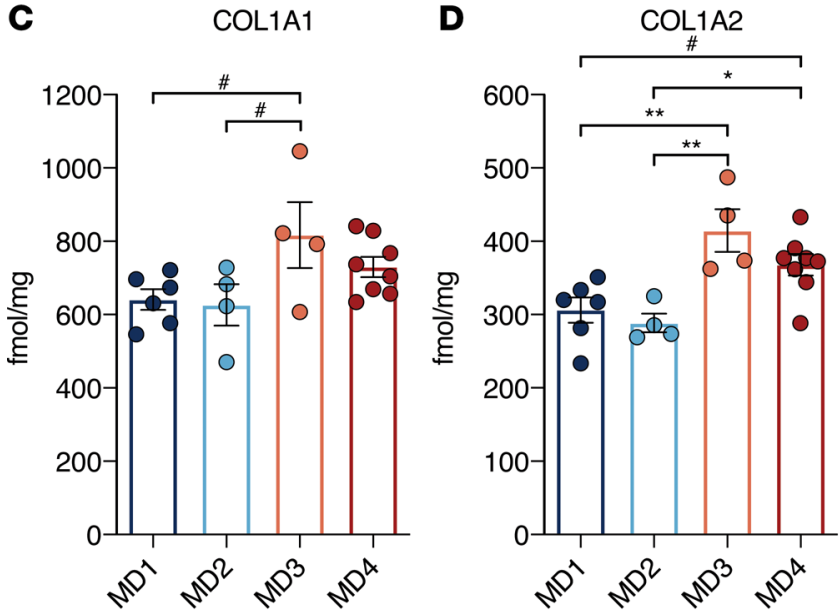

E

COL5A1

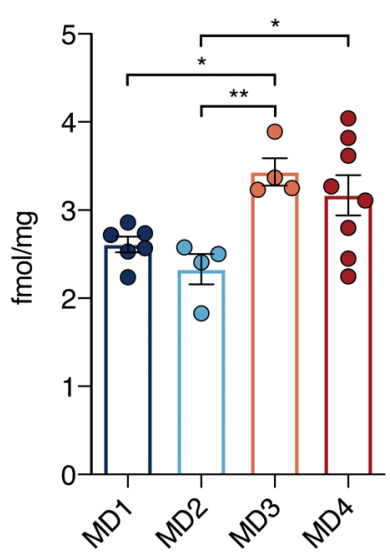

$\mathbf{F}$

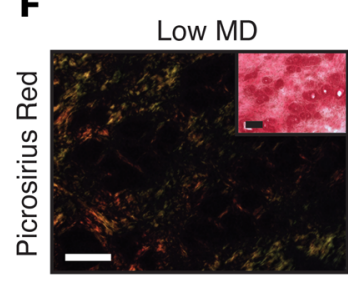

H

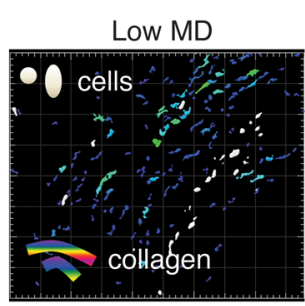

collagen fiber diameter
High MD

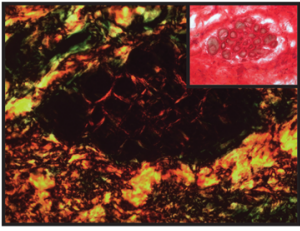

High MD

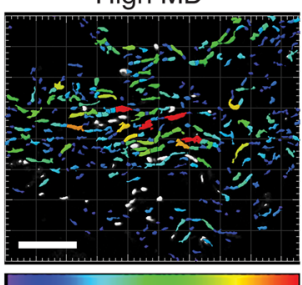

G

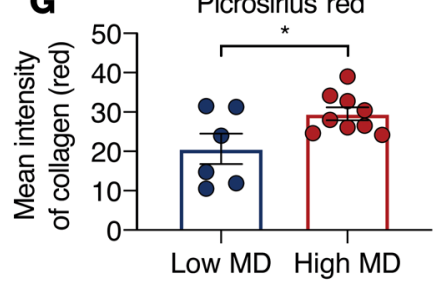

1
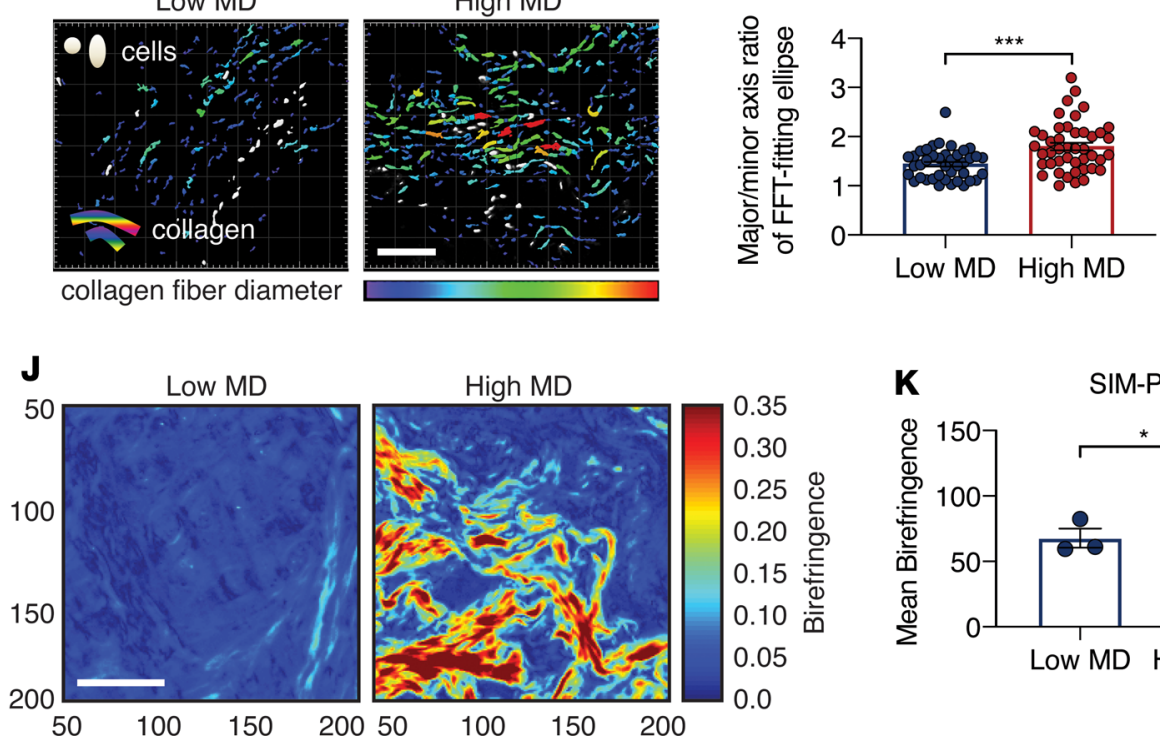

K

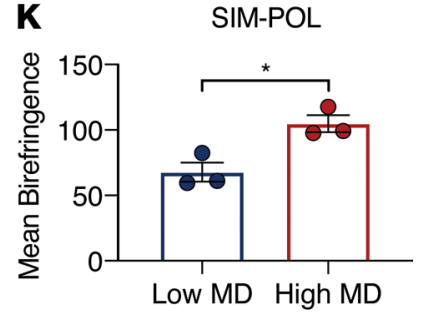

Figure 2. Tissues with high breast density exhibit elevated levels of linear and dense fibrillar collagens.

(A) Trichrome staining of human breast tissue sections to visualize overall collagen matrix abundance (blue) (low MD, $n=4$; high MD, $n=9$ ). Scale bars: $100 \mu \mathrm{m}$ and $50 \mu \mathrm{m}$ (inset). (B) Quantification of trichrome staining represented as percentage positive (blue) area per field of view. (C-E) Quantification of levels for the indicated fibrillar collagens from the proteomic analysis (MD1-MD4; see Figure 1B). (F) Picrosirius red staining of human breast tissue sections and imaging with polarized light to visualize dense collagen fibers (low MD, $n=$ 6; high MD, $n=9$ ). Scale bars: $100 \mu \mathrm{m}$ and $100 \mu \mathrm{m}$ (inset). (G) Quantification of dense collagen (mean intensity of red fibers) visualized by polarized light. (H) Representative images obtained using second harmonic generation (SHC) imaging to visualize the collagen of human breast tissues. Red identifies thicker collagen fibers (bottom). Scale bar: $100 \mu \mathrm{m}$. (I) Quantification of collagen fiber orientation using a model approximation (major/minor axis ratio of a fast Fourier transform-fitting [FFT-fitting] ellipse) (low MD, $n=8$; high MD, $n=$ 8). (J) SIM-POL imaging of breast tissues with MD1 and MD4 to measure tissue birefringence. Blue $\rightarrow$ red color indicates increasing birefringence. Scale bar: $20 \mu \mathrm{m}$. (K) Quantification of SIM-POL imaging showing mean birefringence (low MD, $n=3$, high MD, $n=3$ ). Data are represented as mean \pm SEM. ${ }^{*} P<0.1 ;{ }^{*} P<0.05$; ${ }^{* *} P<0.01$; ${ }^{* *} P<0.001$ by 2 -tailed unpaired Student's $t$ test (G and I), 2-tailed Mann-Whitney $U$ test (B and $\mathbf{K})$, or 1-way ANOVA with Tukey's multiple-comparison test (C-E). 
that the fibrillar collagens in the stroma of the high-MD groups was thicker and more linear than in the low-MD groups (Figure 2, $\mathrm{H}$ and I, and Supplemental Figure 2, A and B). Structured-illumination polarized microscopy (SIM-POL), which concentrates and aligns light images to quantify the birefringence of materials, similarly indicated that the high-MD tissue had significantly more birefringence as compared with the low-MD tissue (Figure 2, J and $\mathrm{K})$. These findings not only confirm prior studies that have reported increased fibrillar collagen in high-MD breast tissue (29), but also suggest there exist substantial structural differences.

We further explored the organization and mechanical phenotype of the fibrillar collagens in the high- and low-MD tissue. Scanning electron microscopy (SEM) revealed that the periductal ECM fibers in the intralobular stroma in the high-MD tissue were not only thicker but were also more densely packed (Figure 3A). Our data suggest that the denser, thicker collagen bundles could reflect the high level of FACIT COL12A1 protein expressed in the high-MD tissue (Figure 3, B and C), which is an ECM protein known to organize type I collagen fibrils into bundles by linking them to the surrounding ECM $(40,41)$. The thickened, linearized collagen bundles in the high-MD tissue could also be due to the greater frequency of contractile $\alpha$-smooth muscle actin-positive ( $\alpha$-SMA-positive) stromal fibroblasts or more ECM remodeling linked to the higher number of fibroblast activation proteinpositive (FAP-positive) stromal fibroblasts we detected in these tissues (Figure 3, D and E, Supplemental Figure 2, C and D, and refs. 42, 43) Moreover, more cross-linked collagen mediated by elevated levels of stromal fibroblast lysyl oxidase (LOX) and lysyl hydroxylase 2 (LH2), which enzymatically induce posttranslational hydroxylation and covalently cross-link lysine residues in the collagens to stabilize and strengthen the fibers, could also account for the thicker, linearized collagens in the high-MD tissue (Figure 3 , F-I, and refs. 31, 44, 45). Regardless of pathogenesis, abundant linearized, oriented, collagen bundles enhance the mechanical property of a collagenous matrix (30-32), as confirmed by atomic force microscopy (AFM) indentation of nonfixed breast tissue, which revealed that indeed the periductal intralobular stroma in the high-MD breast tissue was stiffer (Figure 3, J and K).

In agreement with prior studies linking epithelial and stromal cell density to high MD, and a stiffened ECM with enhanced MEC growth and survival, pathological analysis of H\&E-stained tissue revealed higher epithelial and stromal cell density in the stiffer, high-MD tissue (Figure 3, L and M, and Supplemental Figure 3, A-C). The stiffer, high-MD tissue also exhibited an elevated number of terminal ductal lobular units (TDLUs) with a significantly greater TDLU area and average number of acini per TDLU (Figure 3N and Supplemental Figure 3, D and E). Notably, an increase of percentage in epithelial area in breast tissues correlated positively with elevated stromal ECM stiffness (Figure 30). The findings demonstrate that pathologically normal human breast tissue with high MD not only contains more fibrillar collagen that is thicker and more linearized, but also reveal that this phenotype associates with a stiffer, periductal stroma containing more MECs and stromal fibroblasts.

To more directly interrogate the relationship between collagen architecture, stromal stiffness, and epithelial density, as well as to rule out potentially confounding effects of age, parity, and hormonal status, we collected additional patient samples for analysis of these same organizational and mechanical features within the same breast. We imaged tissue from 2 pathologically confirmed healthy, normal, postmastectomy breasts by whole breast $\mathrm{x}$-ray imaging (Figure 4A). Multiple regions representing high- and low-density regions from within the same postmastectomy breast were then excised and analyzed. Birefringence imaging confirmed that the regions of the microdissected, normal, healthy breast tissue that corresponded to high-density breast tissue contained more aligned stromal components than the tissue regions excised from the low-density regions, echoing the high tissue alignment observed previously in the breast tissue classified as high-MD breast tissue premastectomy (compare Figure 4, B and C, to Figure 2, J and K). AFM indentation confirmed that the periductal stroma in the excised high-density regions of these normal, healthy breast tissues was significantly stiffer than that measured in the regions corresponding to the low-density breast tissue (Figure 4, D and E). Moreover, the epithelial concentration (percentage epithelium) per tissue area was greater in the high-density regions and data analysis revealed that this positively correlated with stromal stiffness, which is consistent with a causal relationship between the 2 parameters (Figure $4, \mathrm{~F}-\mathrm{H}$ ).

miR-203 is repressed by ECM stiffness and decreased in tissues with high MD. miRs are noncoding RNAs with abnormal expression in breast cancer (46). Dysregulation of miRs has been implicated in breast cancer aggression, and miRs are potential biomarkers that could predict risk and progression $(47,48)$. Profiling of miRs expressed in nonmalignant, immortalized human MECs embedded within soft and stiff ECMs and in vivo in mouse mammary tumors treated with and without a LOX inhibitor ( $\beta$-aminopropionitrile, BAPN) to repress collagen cross-linking and prevent stromal stiffening, identified conserved groups of miRs induced and repressed by tissue tension (33). We screened a curated list of these tension-regulated miRs against a list of compiled miRs implicated in breast cancer and interrogated several prospective candidates for tension-modulated expression in the nonmalignant human MECs using qRT-PCR (ref. 49 and Supplemental Table 3). We then experimentally evaluated whether ECM stiffness could modulate the expression of miR18a, miR-203, and miR-149 in cultured murine and human MECs. The expression of the tumor-promoting miR-18a reproducibly increased in nonmalignant human MCF10A MECs plated either on stiff basement membrane-conjugated (BM-conjugated) polyacrylamide (PA) gels or as preassembled acini within 3-dimensional (3D) BM/ collagen gels non-cross-linked (SOFT) or cross-linked and stiffened with L-ribose (STIFF) to achieve previously reported rheometry values (Figure 5, A and B, and ref. 33). Freshly isolated normal, healthy, primary murine mammary organoids embedded within the $3 \mathrm{D}$ ribose-stiffened $\mathrm{BM} /$ collagen gels similarly showed a significant increase in miR-18a expression as compared with the lower levels expressed in the MECs embedded within soft BM/collagen gels (Figure 5C). Nevertheless, although miR-18a stimulates Wnt signaling to promote breast tumor aggression and its expression was found to be significantly increased in the breasts of women with breast cancer (33), we did not detect any increase in miR-18a levels in the normal healthy breast tissue of women with high MD as compared with those with low MD (Figure 5D). By contrast, both miR-203 and miR-149*, 2 miRs previously shown to repress the malignant 

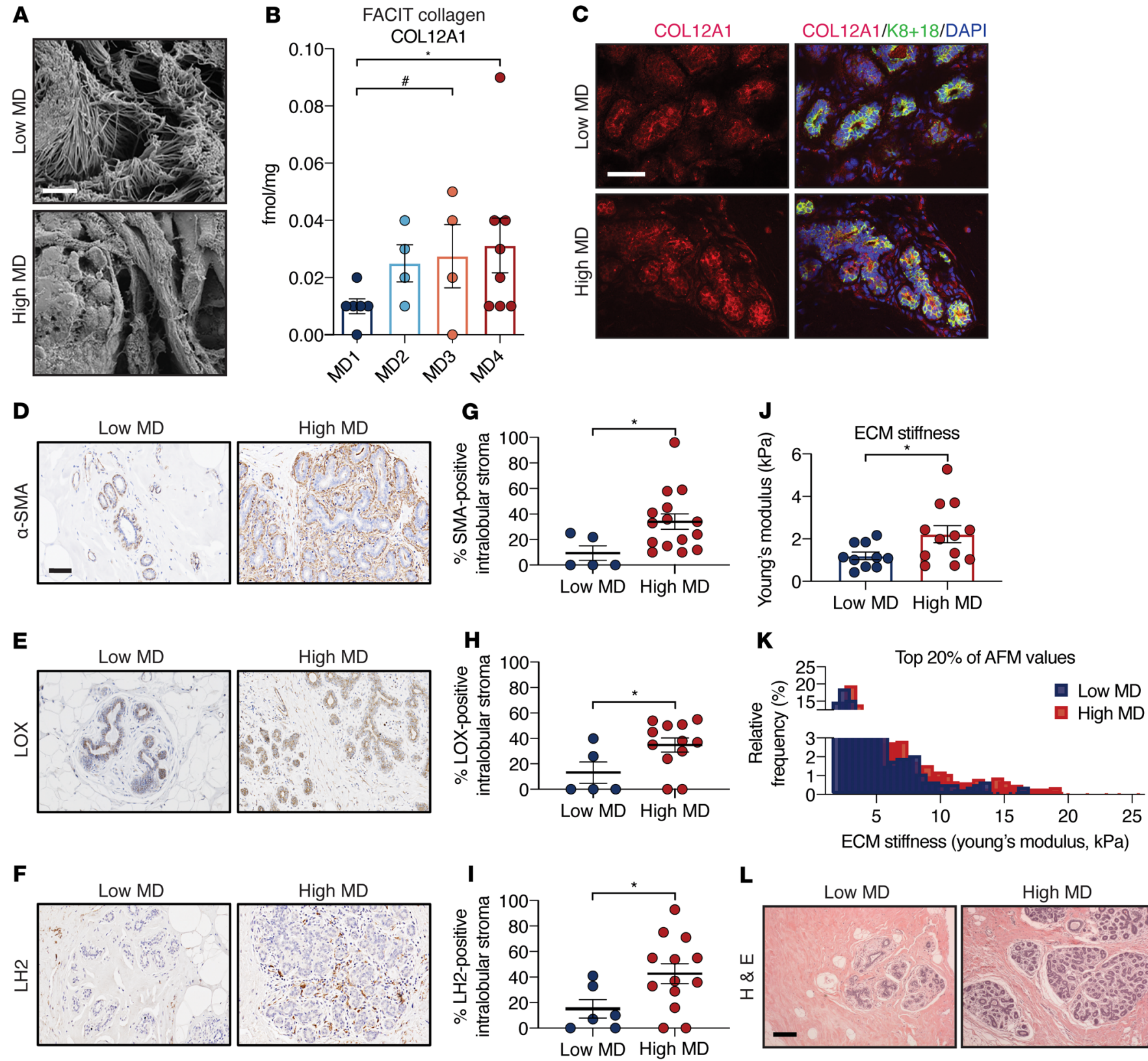

$\mathbf{L}$

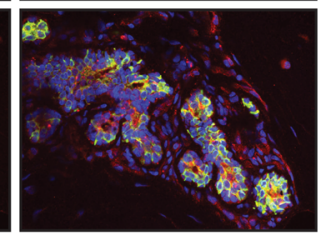

MD
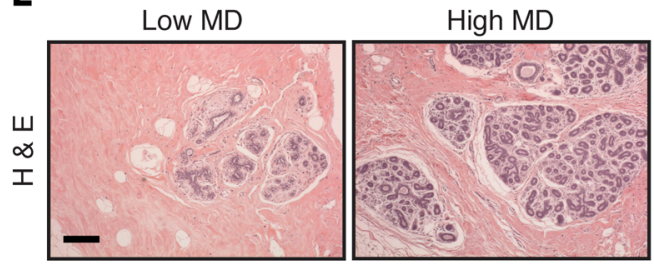

M

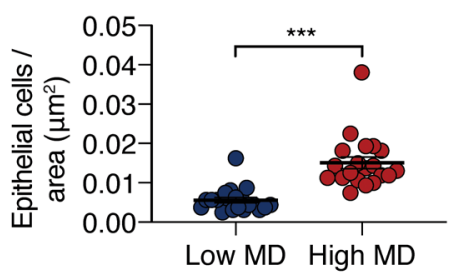

N

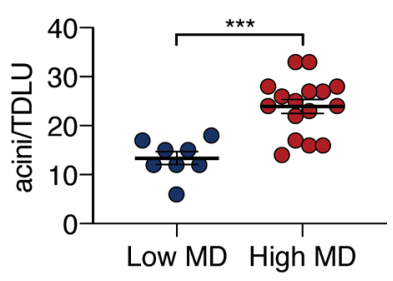

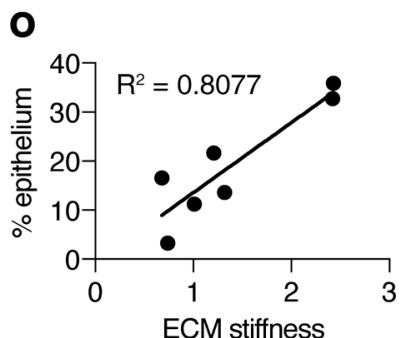


Figure 3. Tissues with high mammographic density are characterized by stiffened collagen fibers, elevated stromal and epithelial density, and expression of collagen cross-linking enzymes. (A) Representative images from scanning electron microscopy (SEM; $n=5$ each for low and high MD). Scale bar: $2 \mu \mathrm{m}$. (B) Quantification of levels for COL12A1 from proteomic analysis (MD1-MD4; see Figure 1). (C) Representative images showing immunofluorescence staining of frozen breast tissue sections with antibodies against keratins 8 and $18(\mathrm{~K} 8+18$, green) and COL12A1 (red) (low $\mathrm{MD}, n=5$, high MD, $n=6$ total). Cell nuclei were stained with 4',6-diamidino-2-phenylindole (DAPI, blue). Scale bar: $50 \mu \mathrm{m}$. (D-F) Immunohistochemical (IHC) staining of human breast tissues using antibodies against $\alpha$-smooth muscle actin ( $\alpha$-SMA) (D), lysyl oxidase (LOX) (E), and lysyl hydroxylase 2 (LH2) (F). Scale bar: $100 \mu \mathrm{m}$. (G-I) Quantification of positive IHC staining for $\mathbf{D}-\mathbf{F}$ as indicated (low MD, $n=5$; high MD, $n=15$ for $\mathbf{G}$; low MD, $n=5$; high MD, $n=12$ for $\mathbf{H}$; low MD, $n=6$; high MD, $n=13$ for I). (J) Quantification of atomic force microscopy (AFM) measurements of the mean elastic modulus (ECM stiffness) of human breast tissues (low MD, $n=10$; high MD, $n=12$ ). (K) A histogram displaying the top $20 \%$ of AFM stiffness values measured in J. (L) Representative images of hematoxylin and eosin-stained (H\&E-stained) human breast tissue sections. (M) Quantification of epithelial density expressed as a percentage of epithelial cell area over total area per field of view (low MD, $n=20$; high MD, $n=20$ ). (N) Quantification of the average number of acini per terminal ductal lobular unit (TDLU) using the same tissues analyzed in Supplemental Figure 3. (0) A correlation plot of measured epithelial cell density (percentage epithelium in a field of view) versus measured ECM stiffness for a selection of human breast tissues $(n=7)$. Data are represented as mean \pm SEM. ${ }^{\#} P<0.1$; ${ }^{*} P<0.05$; ${ }^{* *} P<0.01$; ${ }^{* *} P<0.001$ by 2 -tailed unpaired Student's $t$ test ( $\mathbf{H}-\mathbf{J}$ and $\mathbf{N}$ ), 2-tailed Mann-Whitney $U$ test ( $\mathbf{M}$ and $\mathbf{G}$ ), or Kruskal-Wallis test followed by Dunn's multiple-comparison test (B).

behavior of human breast cancer cells, were repressed both in the MCF10A MECs cultured on the stiff PA gels and as 3D preassembled acini embedded within a ribose-stiffened $\mathrm{BM} /$ collagen gel (Figure 5, E-H). We focused on miR-203 for further analysis because of its prior role in restricting breast cancer progression and its implicated role in breast cancer risk $(38,50-54)$. Consistently, miR-203 expression levels significantly decreased in the freshly isolated primary murine mammary organoids embedded within the ribose-stiffened BM/collagen gels (Figure 5I). Furthermore, qRT-PCR analysis revealed that, on average, miR-203 expression was significantly lower in the normal high-MD breast tissue examined (Figure 5J). In situ hybridization verified that miR-203 expression was substantially lower in the epithelium of the human breast tissue that was classified as high MD, as compared with the high levels detected in the low-MD tissue (Figure 5K). Importantly, miR-203 expression also negatively correlated with the high stromal stiffness quantified in the regions within the normal human breast tissue radiologically classified as high and low MD (Figure 5L). The findings identify the tumor suppressor miR-203 as a tension-regulated molecule that could modulate the elevated lifetime breast cancer risk associated with high MD.

miR-203-mediated targeting of ZNF217 is repressed by ECM stiffness. To clarify whether and how the low levels of miR-203 in the epithelium of the high-density, stiff breast tissue could increase breast cancer risk, we used online tools (miRWalk2.0, miRTarBase) $(55,56)$ to generate a list of predicted miR-203 targets, and identified several previously implicated in breast cancer for further scrutiny (Supplemental Table 4). ZNF217 was selected for further investigation because of its known role in enhancing MEC and breast cancer cell growth, expanding breast progenitor frequency and promoting a mesenchymal transition, and its demonstrated impact on breast cancer progression and aggression (57-59). Furthermore, ZNF217 was previously validated as a miR-203 target in colon cancer cells (60).

We first assessed whether a stiff ECM could modulate the expression of the oncogene ZNF217 in cultured MECs and if this correlated with miR-203 expression. Immunoblot analysis revealed that human immortalized, nonmalignant MCF10A MECs plated on a stiff, BM-conjugated PA gel had higher levels of ZNF217 as compared with the same cells plated on soft PA gels (Figure 6A). We also confirmed that several known ZNF217-influenced gene targets such as SFRP1 and DNMT1 were lower and another, SNAI1, was higher in the same MECs cultured within the stiff ECM gels, indicating that ZNF217 transcriptional regulating activity was also enhanced (Supplemental Figure 4, A-F). We next compared expression levels of Zfp217, the murine homolog of ZNF217, in freshly isolated primary mouse MEC organoids embedded within soft BM/collagen gels (non-cross-linked) as compared with those that were embedded within stiff BM/collagen gels (L-ribose cross-linked). qRT-PCR analysis confirmed that $Z f p 217$ was indeed significantly increased in the organoids embedded within the stiffened $\mathrm{BM} /$ collagen gels as compared with within the softer $\mathrm{BM} /$ collagen gels (Figure 6B). Correlation analysis revealed an inverse relationship between high Zfp217 and low miR-203 expression in the same acini (Figure 6C).

To directly test for a causal relationship between tensionregulated miR-203 and ZNF217 expression and activity, we generated nonmalignant human MCF10A MECs that overexpressed miR-203, and MECs expressing either a scrambled or a miR-203targeting antagomir. Immunoblot analysis showed that the high expression of ZNF217 detected in the MECs cultured on stiff 2D PA gels $(6 \mathrm{kPa})$ could be overridden either by increasing miR-203 levels to reduce ZNF217, or by increasing ZNF217 levels using the miR-203 antagomir (Figure 6A). Moreover, although the MEC acini that were embedded within ribose cross-linked, stiffened $\mathrm{BM} /$ collagen gels that expressed a control miR (CTL) had high levels of ZNF217, those that expressed high miR-203 (203 ${ }^{\mathrm{OE}}$ ) had significantly lower levels of ZNF217 (Figure 6, D and E). Consistently, knocking down miR-203 (203 ${ }^{\mathrm{KD}}$ ) in the MCF10A MEC acini increased ZNF217 expression, even when the acini were grown within the soft $\mathrm{BM} /$ collagen hydrogels (Figure 6, F and G). Increasing, or decreasing, ZNF217 levels by modulating miR-203 in MECs within soft or ribose cross-linked, stiffened $\mathrm{BM} /$ collagen gels, also altered the expression of ZNF217 targets (Supplemental Figure 4, A-F). We observed similar changes in the activity of the known ZNF217 target PI3K/Akt in the MECs with altered ZNF217 levels, as revealed by immunoblot analysis of phosphorylated Akt substrates (Figure 6A).

ZNF217 activates PI3K/Akt to promote MEC proliferation $(57,61,62)$. Immunofluorescence staining for the proliferative marker phosphorylated histone $\mathrm{H} 3$ (p-HH3) as well as phosphorylated Akt substrates revealed that those MEC acini expressing high ZNF217 and low miR-203 were also the most proliferative and had the highest Akt activity (Figure 6, H-M, and Supplemental Figure 4, G and H). Reducing ZNF217 by overexpressing miR-203 simultaneously repressed cell growth and reduced the level of phosphorylated Akt substrates as detected by immunofluorescence staining (Figure 6, H, J, and L, and Supplemental 
SIM-POL Birefringence
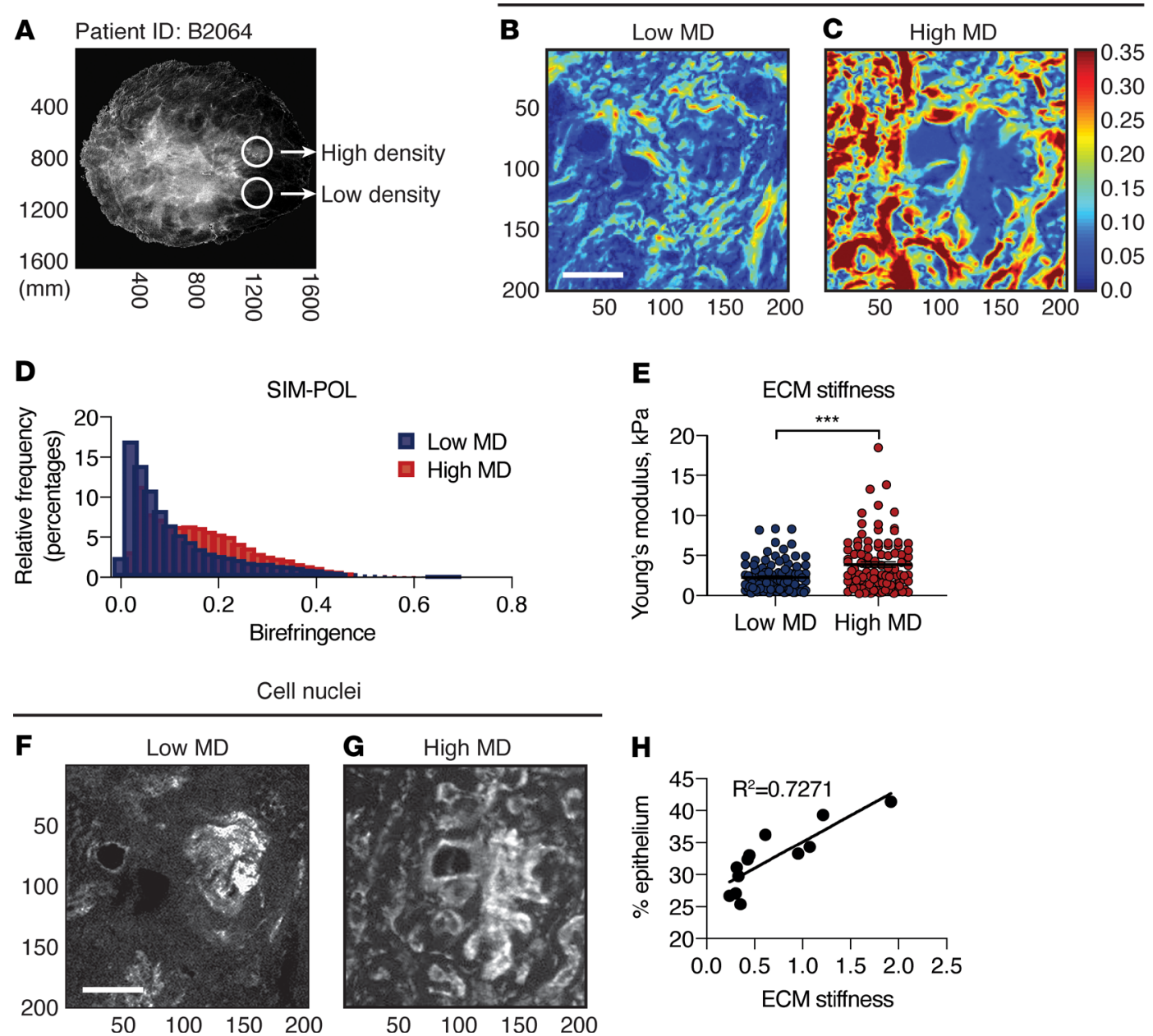

Figure 4. Regions of high mammographic density within the same breast correlate with increased ECM stiffness and epithelial density. (A) Mammography of a whole breast with demarcations highlighting regions of high and low density used for subsequent analysis. (B) SIM-POL imaging of the lowdensity region selected in $\mathbf{A}$ to measure tissue birefringence and approximate ECM tension. Blue $\rightarrow$ red indicates increasing birefringence. Scale bar: $20 \mu \mathrm{m}$. (C) The same as in $\mathbf{B}$ for the high-density region selected in $\mathbf{A}$. (D) A representative histogram of SIM-POL measurements for the tissue regions selected in A (low MD, $n=5$; high MD, $n=5$ total). (E) Quantification of AFM measurements of human breast tissues isolated from multiple regions of high and low density (low MD, $n=10$; high MD, $n=10$ ) from within the same breast. Individual ECM stiffness measurements from several regions are plotted ( $n=100$ each for low and high MD). (F) The low-density region from $\mathbf{A}$ was stained with propidium iodide to permit visualization of cell density by fluorescence. Scale bar: $20 \mu \mathrm{m}$. (G) The same as in $\mathbf{F}$ for the high-density region selected in $\mathbf{A}$. (H) A correlation plot of epithelial cell density (percentage epithelium, percentage of propidium iodide stained epithelial nuclei per field of view) versus ECM stiffness from human breast tissues $(n=3)$ with multiple regions of low and high MD from within the same breast $(n=12)$. Data are represented as mean \pm SEM. ${ }^{* * *} P<0.001$ by 2 -tailed Mann-Whitney $U$ test $(E)$.

Figure 4G). By comparison, expressing the antagomir of miR-203 concomitantly increased ZNF217 expression, MEC proliferation, and Akt activity (Figure 6, I, K, and M, and Supplemental Figure $4 \mathrm{H})$. In agreement with prior data supporting a role for miR-203 and the ZNF217 target PI3K/Akt in MEC invasion (38), MEC acini expressing low miR-203 and high ZNF217 were more invasive, as compared with control MEC acini cultured in soft BM/ collagen gels, and MEC acini cultured in stiff $\mathrm{BM} /$ collagen gels in which ZNF217 levels were reduced by overexpressing miR-203 (Supplemental Figure 5, A-D). To further implicate ZNF217 in mediating the phenotypes of proliferation, Akt activity and invasion, we generated an shRNA-mediated knockdown of ZNF217 in the MCF10A MECs expressing the scrambled (Scr) or miR203-targeting (203 $\left.{ }^{\mathrm{KD}}\right)$ antagomirs. Immunoblot analysis was used to validate 3 shRNAs (F7, F9, and G4) targeting ZNF217 for knockdown compared with cells expressing a vector control (VC) (Supplemental Figure 6A). The shRNA G4 exhibited the most efficient knockdown and was used for subsequent analyses of MCF10A MECs cultured in BM/collagen hydrogels with or without L-ribose-mediated cross-linking and stiffening. ZNF217 knockdown abrogated the increase in proliferation and Akt activity induced by a stiff ECM, as assessed by immunofluorescence staining for $\mathrm{p}-\mathrm{HH} 3$ and phosphorylated Akt substrates in both MCF10A MECs expressing the scrambled and miR-203targeting antagomirs (Supplemental Figure 6, B-I). These findings demonstrate that a stiff ECM can enhance MEC proliferation and invasion by reducing levels of miR-203 to elevate expression of the oncogene ZNF217 and thereafter to increase PI3K/Akt activity.

We next asked whether the increased expression of ZNF217 stimulated by a stiff ECM also promoted MEC proliferation and 

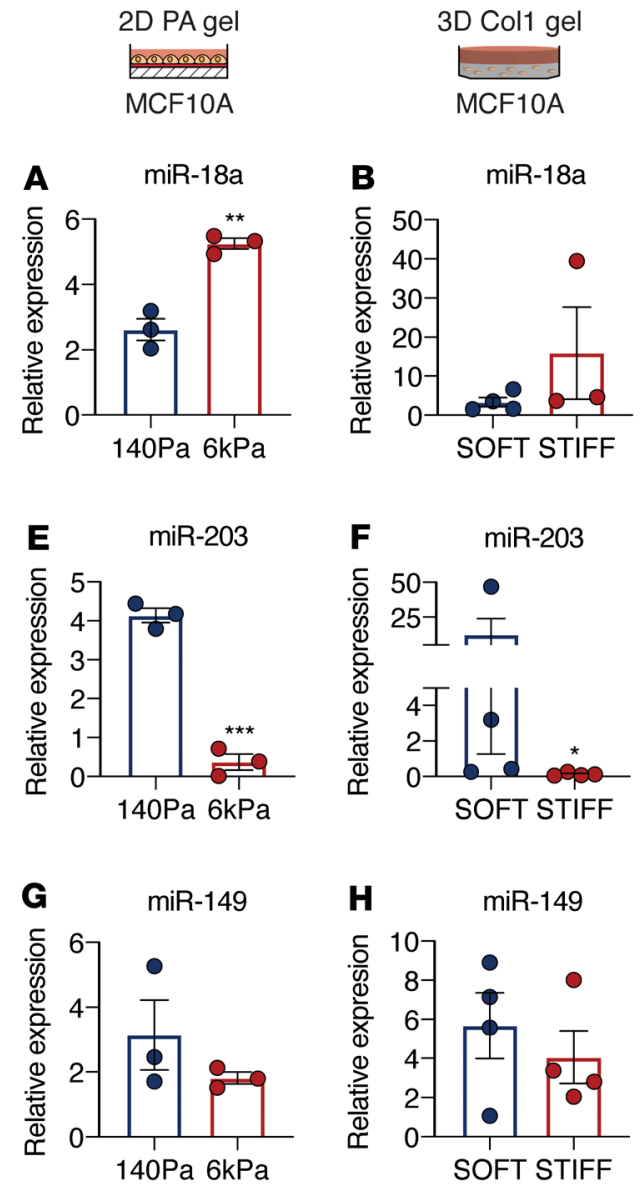
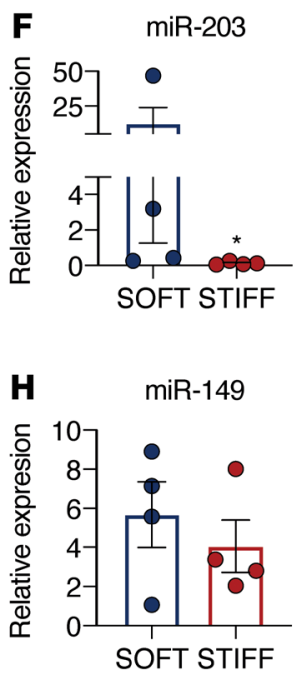
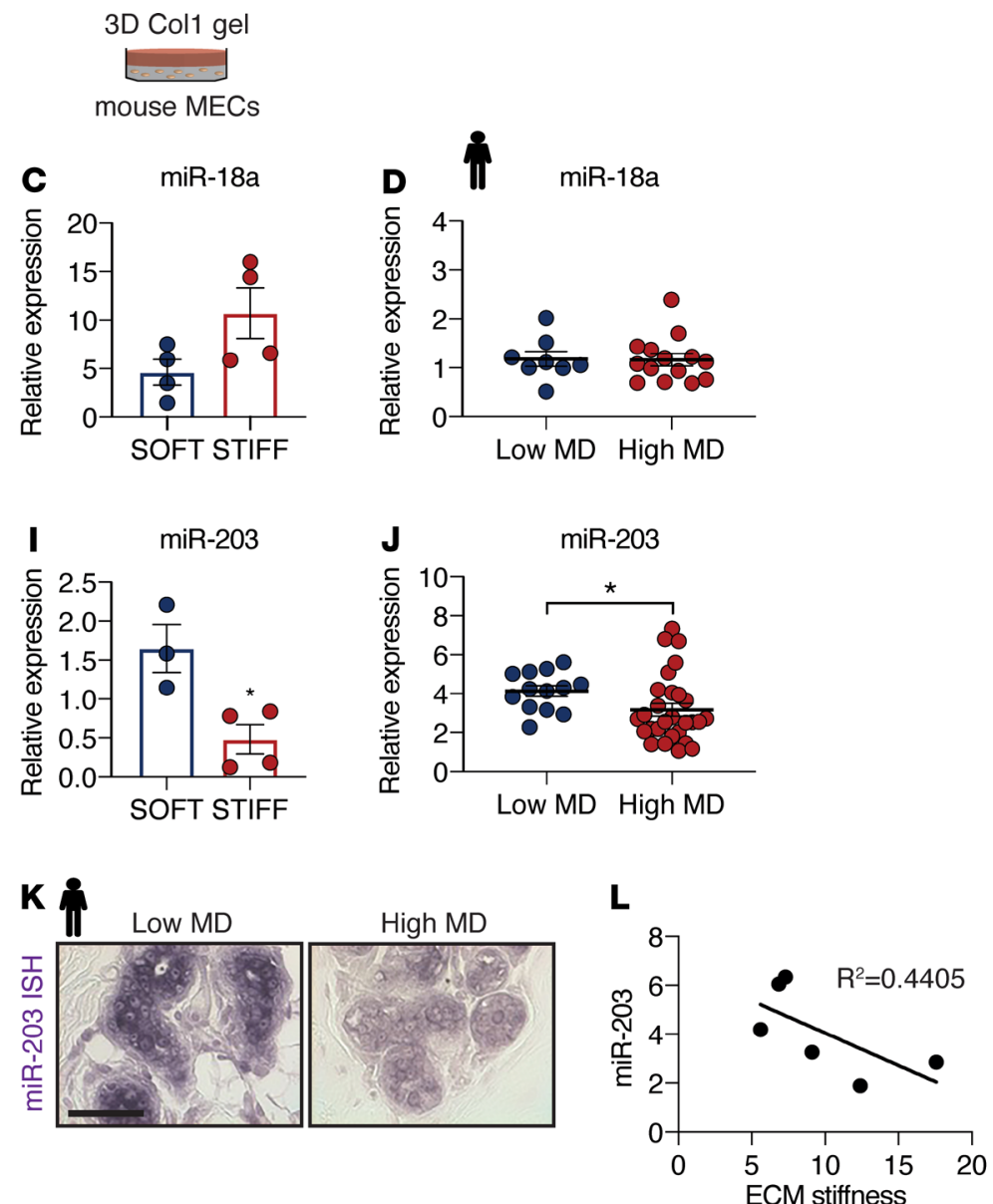

Figure 5. ECM stiffness represses the expression of miR-203, which exhibits reduced expression in tissues with high mammographic density. (A, E, and G) qRT-PCR analysis for the indicated microRNAs using RNA isolated from MCF10A cells cultured on basement membrane-conjugated (BM-conjugated) polyacrylamide (PA) gels of varying stiffness ( $140 \mathrm{~Pa}$ and $6 \mathrm{kPa})$. Results are normalized to U6 RNA and plotted relative to the $140-\mathrm{Pa}$ condition ( $n=3$ replicates each for $140 \mathrm{~Pa}$ and $6 \mathrm{kPa}$ ). (B, F, and $\mathbf{H}$ ) qRT-PCR analysis for the indicated microRNAs using RNA isolated from MCF10A acini cultured in SOFT (non-cross-linked) or STIFF (L-ribose cross-linked) BM/collagen gels. Results are normalized to U6 RNA and plotted relative to the SOFT condition ( $n=3-4$ replicates for SOFT and STIFF). (C and I) qRT-PCR analysis for the indicated microRNAs using RNA isolated from mouse mammary epithelial acini cultured as in B. Results are normalized to U6 RNA and plotted relative to the SOFT condition ( $n=3-4$ replicates for SOFT and STIFF). (D and J) qRT-PCR analysis for the indicated microRNAs using RNA isolated from human breast tissues with low and high MD. Results are normalized to U6 RNA and plotted as individual data points (low MD, $n=8$, high $\mathrm{MD}, n=14$ for $\mathbf{D}$; low MD, $n=13$, high $\mathrm{MD}, n=28$ for J). (K) Representative images of in situ hybridization (ISH) analysis for miR-203 expression (purple) in breast tissues (low MD, $n=3$; high MD, $n=3$ total). Scale bar: $50 \mu \mathrm{m}$. (L) Correlation between relative miR-203 expression levels and ECM stiffness for human breast tissue specimens $(n=6)$. Data are represented as mean \pm SEM. ${ }^{*} P<0.05 ;{ }^{* *} P<0.01 ;{ }^{* * *} P<0.001$ by 2-tailed unpaired Student's $t$ test (A, E, and I) or 2-tailed Mann-Whitney $U$ test (F and $\mathbf{J})$.

increased MEC density in the mammary gland in vivo. We studied this relationship in the Colla1 $1^{\text {tmIIae }}$ mouse, which has been used previously as a murine model of collagen density $(35,63)$. Heterozygous Col1a1 ${ }^{\text {tmIJae }}\left(\mathrm{COL}^{+-}\right)$mice carry a transgene with mutations in a highly conserved MMP cleavage domain of Colla1 that leads to accumulation of collagen surrounding the mammary epithelial ducts. The elevated collagen levels in the $\mathrm{COL}^{+/-}$mouse result in an increase in the tensile properties of the mammary gland tissue that mimic the stiffer breast stroma we quantified in the high-MD human breast stroma (ref. 35 and Figure 3, J and K). Consistent with an association between high collagen density, stromal stiffness, and elevated risk of malignancy, we observed an almost 2-fold increase in p-HH3 staining in the MECs of the ducts of 10-week-old $\mathrm{COL}^{+/}$mice (Figure 7, A and B), that qRT-PCR revealed correlated with reduced levels of miR-203 (Figure 7C). Immunohistochemical staining also detected more than 2-fold higher nuclear Zfp217 in the MECs in the $\mathrm{COL}^{+/}$mice (Figure 7, D and E), and higher levels of phosphorylated Akt substrates, presumably reflecting elevated activity of the Zfp217 target Akt (Figure 7, F and G). Consistently, when the $\mathrm{COL}^{+/}$mice were treated with the ZNF217/Akt inhibitor triciribine, the level of phosphorylated Akt substrate and p-HH3 immunohistochemical staining were significantly diminished in the mammary epithelium (Figure 7, A, B, F, and G). The findings are consistent with prior studies, which showed that when Colla1 $1^{\text {thlae }}$ mice were crossed with PyMT mice they exhibited accelerated mammary tumor progression (35). The data obtained in the Col1a1 ${ }^{\text {tmIJae }}$ mice suggest that the reduced miR-203 and high ZNF217 and epithelial proliferation observed in the breast tissue of the women with high MD is likely mediated by the increased collagen density and stromal stiffness. The findings could also explain 
A

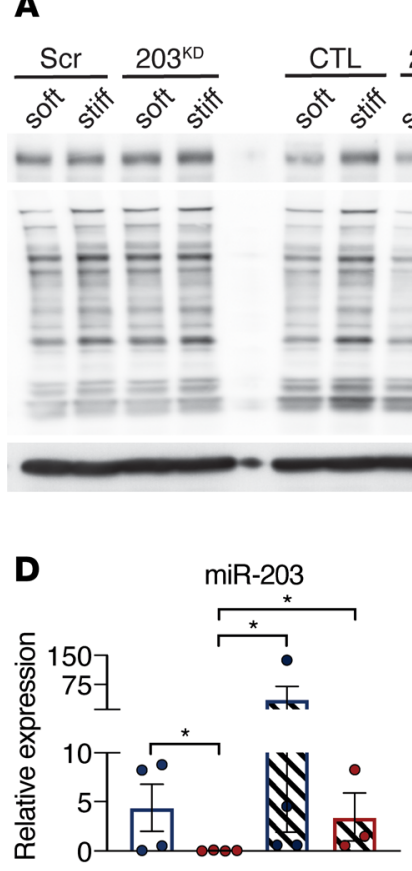

$\mathbf{F}$

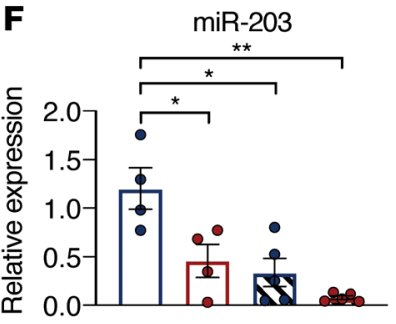

2D PA gel

r

MCF10A

ZNF217

Phosphorylated Akt substrate
B
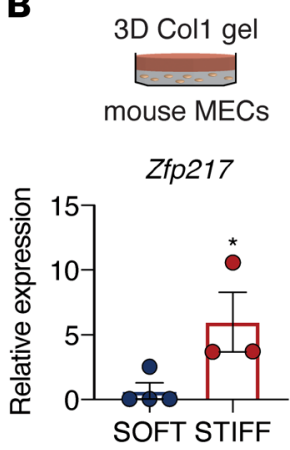

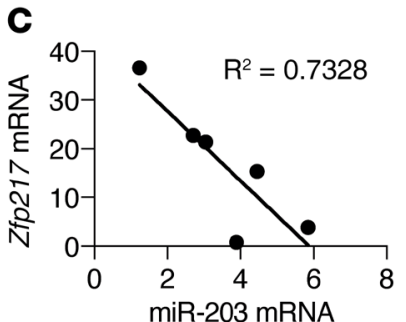

$\beta$-actin

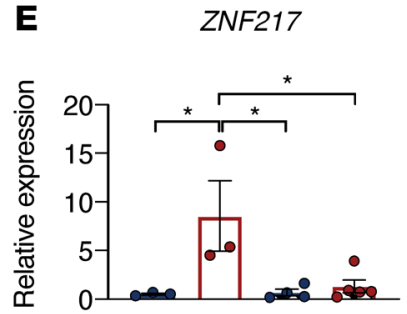

H Phospho-Histone H3

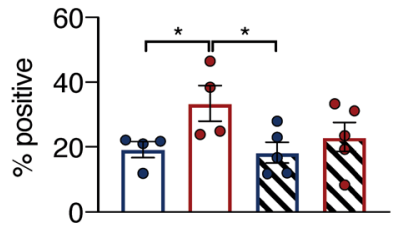

$\square$ SOFT CTL $\square$ STIFF CTL II SOFT $203^{\circ}$ III STIFF $203^{\circ}$
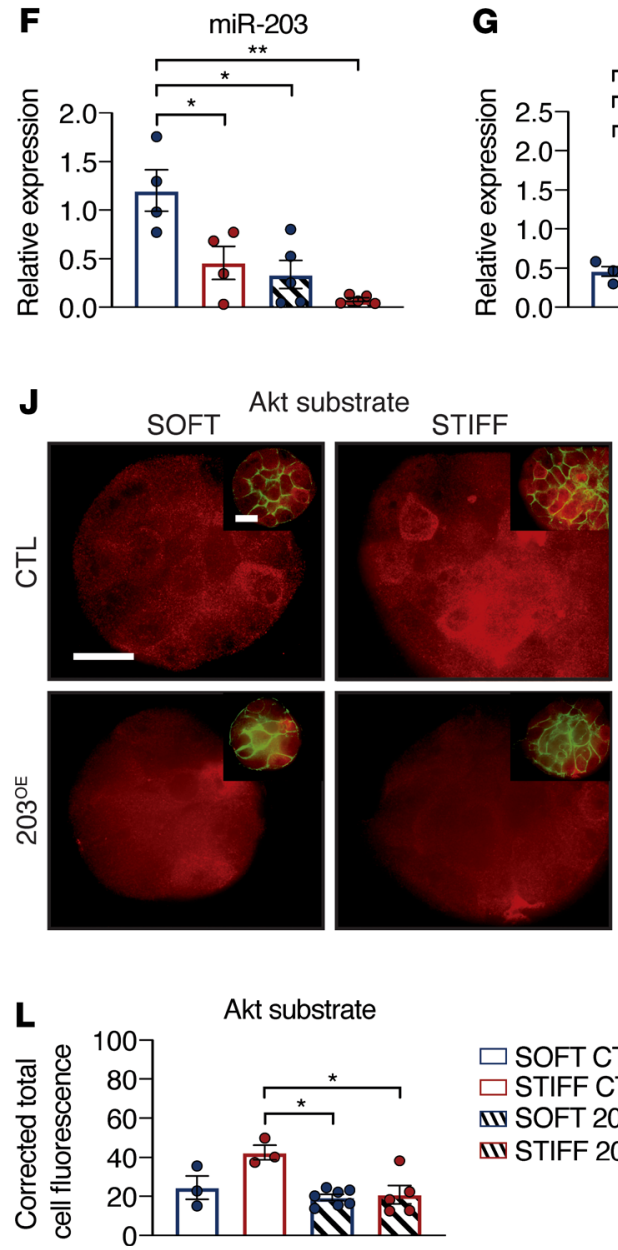

$\square$ SOFT CTL

$\square$ STIFF CTL

II SOFT $203^{\circ}$

III STIFF $203^{\circ}$

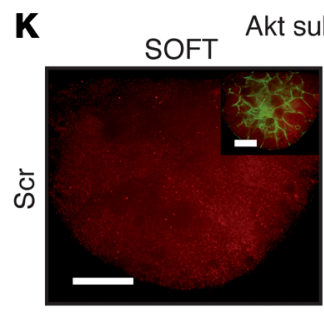

I Phospho-Histone $\mathrm{H} 3$

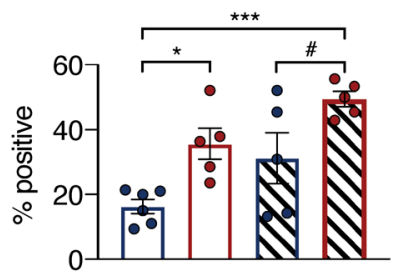

$\square$ SOFT Scr

$\square$ STIFF Scr IIv SOFT $203^{\mathrm{KD}}$

III STIFF $203^{\mathrm{KD}}$

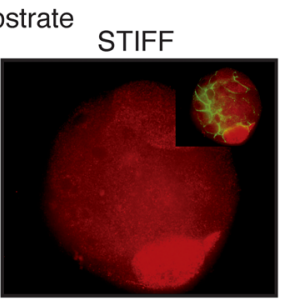

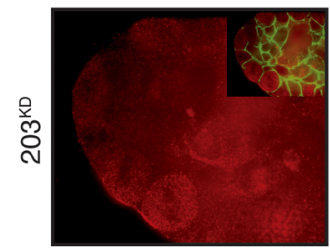
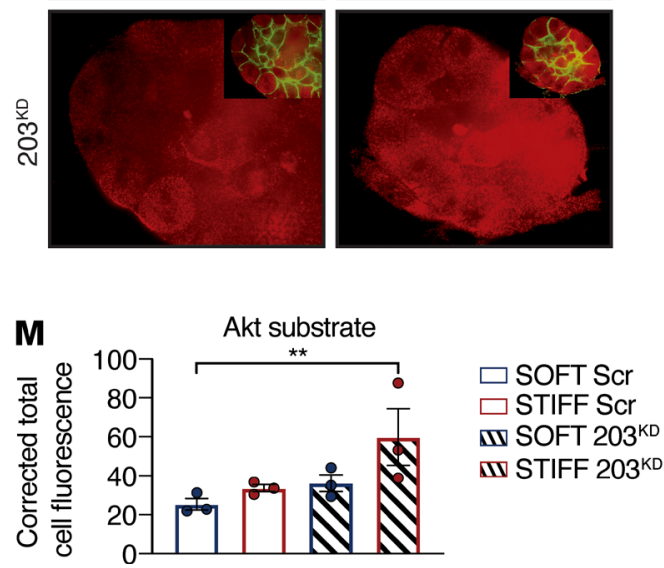

ठํํ워 
Figure 6. The proliferative factor ZNF217 is regulated by ECM stiffness in a miR-203-dependent manner and high ZNF217 levels correlate with increased MEC proliferation and Akt activity. (A) MCF10A acini overexpressing a scrambled nontargeting antagomir (Scr) or an antagomir targeting miR-203 (203 ${ }^{\mathrm{KD}}$ ) (lanes 1-4) or MCF10A acini overexpressing a control microRNA (CTL) or miR-203 (203 ${ }^{\circ \mathrm{E}}$ ) (lanes 5-8) were cultured on soft $(140 \mathrm{~Pa})$ or stiff $(6 \mathrm{kPa}) \mathrm{PA}$ gels conjugated with $\mathrm{BM}$. Cells were cultured for 24 hours and harvested for immunoblot analysis using antibodies against ZNF217, phosphorylated Akt substrate, or $\beta$-actin (representative of 2 experiments). (B) qRT-PCR analysis for Zfp217 using RNA isolated from mouse mammary epithelial acini cultured as in Figure 5B. Results are normalized to Gapdh levels and plotted relative to the SOFT condition (SOFT, $n=4$, STIFF, $n=3$ ). (C) A correlation between measured Zfp217 levels from B with miR-203 levels from Figure 5I. (D) qRT-PCR analysis for miR-203 using RNA isolated from CTL or $203^{\circ \mathrm{EE}}$ MCF10A acini and cultured as in B. Results are normalized to U6 RNA and plotted relative to the SOFT CTL condition ( $n=3-4$ replicates). (E) qRT-PCR analysis for ZNF217 using RNA isolated from the same MCF10A acini cultured as in $\mathbf{D}$. Results are normalized to 185 RNA and plotted relative to the SOFT CTL condition ( $n=$ 3-5 replicates). (F) qRT-PCR analysis for miR-203 using RNA isolated from Scr or $203^{\mathrm{KD}}$ MCF10A acini and cultured as in B. Results are normalized as in D ( $n=4-5$ replicates). (C) qRT-PCR analysis for ZNF217 using RNA isolated from the same MCF10A acini cultured as in F. Results are normalized as in $\mathbf{E}$ ( $n=4$ replicates). (H) MCF10A acini manipulated and cultured as in D were fixed and stained by immunofluorescence with antibodies against phosphorylated histone $\mathrm{H3}$ (phospho-histone $\mathrm{H3}$, red) and phalloidin-488 (green). Quantification of the average number of phospho-histone H3positive nuclei expressed as a percentage of total nuclei per field of view ( $n=4-5$ replicates). (I) MCF10A acini manipulated and cultured as in $\mathbf{F}$ were analyzed and quantified as in $\mathbf{H}$ ( $n=5-6$ replicates). (J) MCF10A acini manipulated and cultured as in $\mathbf{D}$ were fixed and stained by immunofluorescence with antibodies against phosphorylated Akt substrate (red) and phalloidin-488 (green). Representative images are presented. (K) Representative images of MCF10A acini manipulated and cultured as in F and processed as in J. (L) Quantification of the average corrected total cell fluorescence per field of view for MCF10A acini prepared as in J ( $n=3-7$ replicates). (M) Quantification of the average corrected total cell fluorescence per field of view for MCF10A acini prepared as in $\mathbf{K}$ ( $n=3$ replicates). Data are represented as mean $\pm \mathrm{SEM}$. ${ }^{\#} P<0.1 ;{ }^{*} P<0.05 ;{ }^{* *} P<0.01$; ${ }^{* *} P<$ 0.001 by 2-tailed unpaired Student's $t$ test (B), 1-way ANOVA with Tukey's multiple-comparison test (E, F, I, L, and $\mathbf{M}$ ), or Kruskal-Wallis test followed by Dunn's multiple-comparison test (D, $\mathbf{G}$, and $\mathbf{H})$.

why the breast cancers that develop in women with high MD are often more aggressive (64-66).

Mammary tissues with high collagen density display elevated ZNF217 expression, epithelial proliferation, and epithelial density. We next examined the relationship between MD, stromal stiffness, epithelial proliferation, and miR-203-regulated ZNF217 in human breast tissue. Immunohistochemical staining revealed a significant increase in nuclear staining for ZNF217 in the MECs within the high-MD breast tissue, and quantitative analysis revealed that this staining correlated positively with high nuclear staining for $\mathrm{p}-\mathrm{HH} 3$ (Figure 8, A-D). In agreement with our findings in the $\mathrm{COL}^{+/-}$mice, ZNF217 protein levels correlated positively with epithelial density (percentage epithelium) and negatively with miR-203 expression (Figure 8, E and F). The findings suggest that a stiffer, high-density breast stroma could increase epithelial density to enhance breast cancer risk by elevating levels of ZNF217 to foster MEC proliferation.

miRs including miR-203 have many targets, including gene products that both promote and inhibit transformation and tumor aggression (e.g., refs. 38, 50, 60, 67). Accordingly, the risk of breast cancer induced by high-MD-associated, tensioninduced miR-203 loss should reflect the combined effect of its pro- and antitumor molecular targets on the breast tissue's phenotype. Consistently, we showed that a stiff ECM reduces miR203 in murine MECs and a nonmalignant human MEC cell line to induce the tumor suppressor ROBO1 (38). High levels of ROBO1 reduce MEC invasion in culture and blunt branching morphogenesis of the murine mammary gland in vivo (38). qRT-PCR analysis revealed that $R O B O 1$ levels were significantly higher in the breast tissue from the women with high MD, consistent with a compensatory ROBO1-dependent repression of malignancy in these breast tissue epithelia (Figure 8G). Critically however, analysis of the ZNF217/ROBO1 expression ratio in individual breast tissue specimens revealed that 4 out of 14 of the high-MD tissues had a $Z N F 217 / R O B O 1$ expression ratio that exceeded one, and that 2 of the biospecimens showed a more than 5- to 10-fold disproportionately elevated ZNF217/ROBO1 ratio (Figure $8 \mathrm{H})$. The findings not only suggest that the collagen-dense, stiff stroma in the high-MD breast could expand the mammary epithelium to increase breast cancer risk, but that it simultaneously increases expression of key tumor suppressors and oncogenes, the ratio of which will ultimately dictate predisposition of the epithelium to malignant transformation. The findings are all the more compelling given prior evidence showing that $R O B O 1$ is methylated in over $45 \%$ of human breast tumors (68-71). As such, the stiffer stroma could be instrumental in establishing the biological basis for many of the phenotypes classically associated with high MD and suggest that the paradigm may impart a general qualitatively greater risk for the development of breast cancer by modulating expression of key oncogenes and tumor suppressors. If true, treatments aimed at inhibiting tension-induced oncogene expression would constitute novel chemoprevention modalities, and assessment of methylation and/or mutations in tension-modulated tumor suppressors could help to identify patients at higher risk for developing breast tumors.

\section{Discussion}

Our studies established a causal association between collagen density, breast stroma stiffness, and epithelial density. We identified a tension-mediated mechanism that both fosters MEC growth and predisposes the epithelium to transformation by regulating levels of a key oncogene and tumor suppressor. Experimental data revealed that a stiff ECM represses levels of the tumor suppressor miR-203, whose tension-induced loss increases expression of the oncogene ZNF217 to increase epithelial density by promoting epithelial proliferation. The stiff, high-MD breast tissue expressed elevated levels of ZNF217 that correlated with lower miR-203 and higher epithelial proliferation and density. The results agree with previous data showing that high-MD breast tissue contains a higher density of MECs (7-13). Importantly, our studies indicated that there is a significant correlation between MEC proliferation, epithelial density, and MD (Figure 4), consistent with some prior published reports but contradictory to others $(9,10,13,72-76)$. We analyzed high- and low-MD tissue obtained exclusively from premenopausal women (Supplemental Table 1) where estrous cycling stimulates MEC proliferation. Importantly, 
A

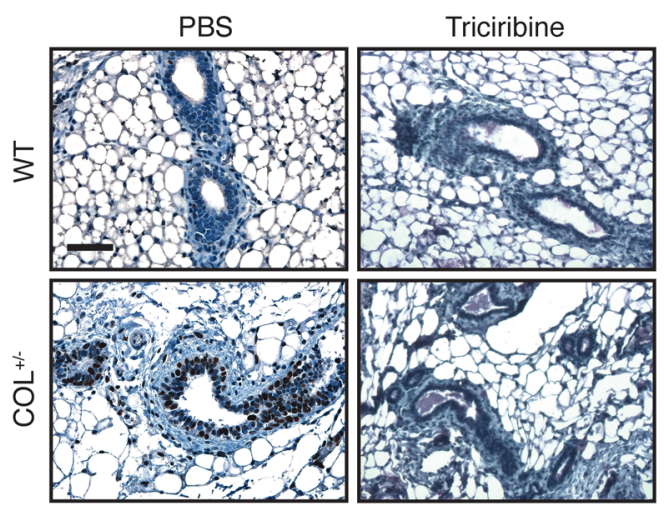

B

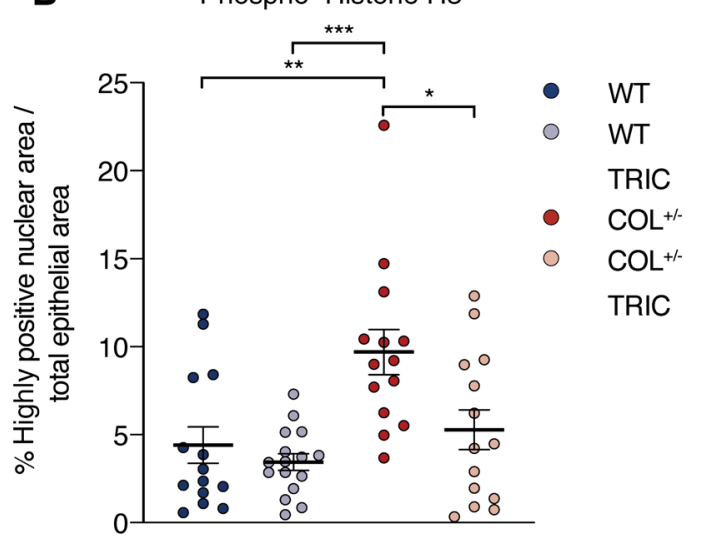

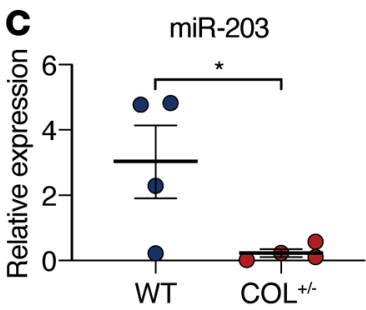

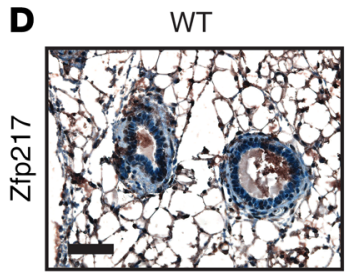

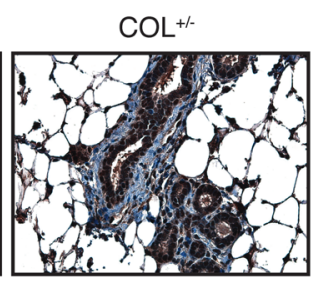

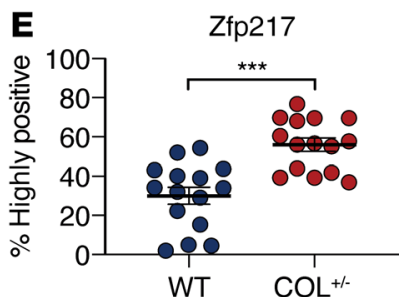

WT
$\mathbf{F}$

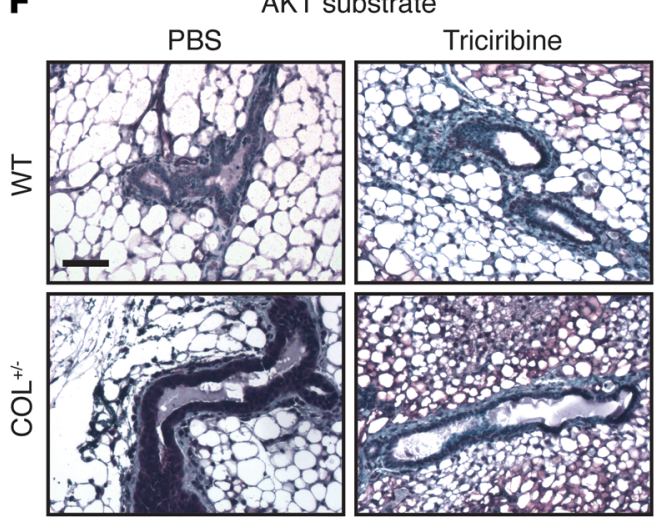

G

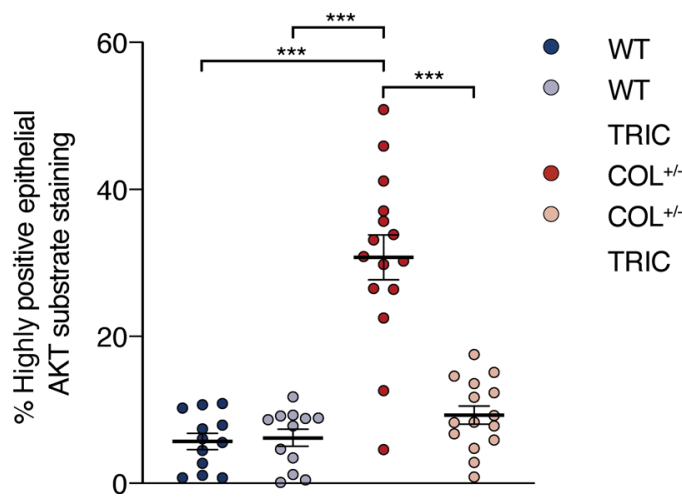

Figure 7. ZNF217 inhibition with triciribine abrogates stiff collagen matrix-induced mammary epithelial cell proliferation and Akt activity in vivo. (A) Immunohistochemical (IHC) staining of paraffin sections from the mammary glands of heterozygous Col7a $7^{\text {tmllae }}\left(\mathrm{COL}^{+/-} ; n=3\right)$ and WT $(n=3)$ mice using a phospho-histone H3-specific antibody. Selected mice were treated with the ZNF217/Akt inhibitor triciribine (TRIC; $n=3$ each for WT and COL ${ }^{+/}$ mice). Scale bar: $50 \mu \mathrm{m}$. (B) Quantification of positive phospho-histone H3 staining from A expressed as the percentage of highly positive nuclei area per total epithelial area $(n=14-16)$. (C) qRT-PCR analysis for miR-203 using RNA isolated from the mammary glands of 10 -week-old COL ${ }^{+/-}$mice and agematched WT counterparts. Results are normalized to U6 RNA levels and plotted relative to WT $(n=4)$. (D) IHC staining of paraffin sections as in $\mathbf{A}$ using a ZNF217-specific antibody. Scale bar: $50 \mu \mathrm{m}$. (E) Quantification of positive ZNF217 staining from $\mathbf{D}$ expressed as the percentage of high positive staining in MECs $(n=15)$. (F) IHC staining of paraffin sections as in $\mathbf{A}$ using a phosphorylated Akt substrate-specific antibody. Selected mice were treated with triciribine as in A. Scale bar: $50 \mu \mathrm{m}$. (C) Quantification of positive phosphorylated Akt substrate staining from $\mathbf{F}$ expressed as the percentage of high positive staining in MECs $(n=12-15)$. Data are represented as mean \pm SEM. ${ }^{*} P<0.05$; ${ }^{* *} P<0.01 ;{ }^{* *} P<0.001$ by 2-tailed unpaired Student's $t$ test (C and E) or Kruskal-Wallis test followed by Dunn's multiple-comparison test (B and $\mathbf{G})$.

larger cohort studies that included pre- and postmenopausal breast tissue also established associations between MD and epithelial density but did not observe a correlation with proliferation, likely because the postmenopausal breast is essentially quiescent $(9,13,74-76)$. Accordingly, our data confirm that a correlation exists between high MD and epithelial density and, furthermore, suggest this might be due to higher overall proliferation of the premenopausal mammary epithelium. The findings thus predict that having more epithelial targets for oncogenesis is likely a major contributing factor that would proportionately increase the risk of breast cancer in these women.

Our rigorous quantitative proteomic analysis of the insoluble ECM and imaging assessment of predominantly healthy human MD1, MD2, MD3, and MD4 breast tissue confirm prior immunostaining studies reporting increased fibrillar collagen and proteoglycans in the high-MD breast tissue (13, 27-29, 77-80). The findings also extend prior studies to identify unique structural collagens implicated in collagen bundling such as COL12A1, and 

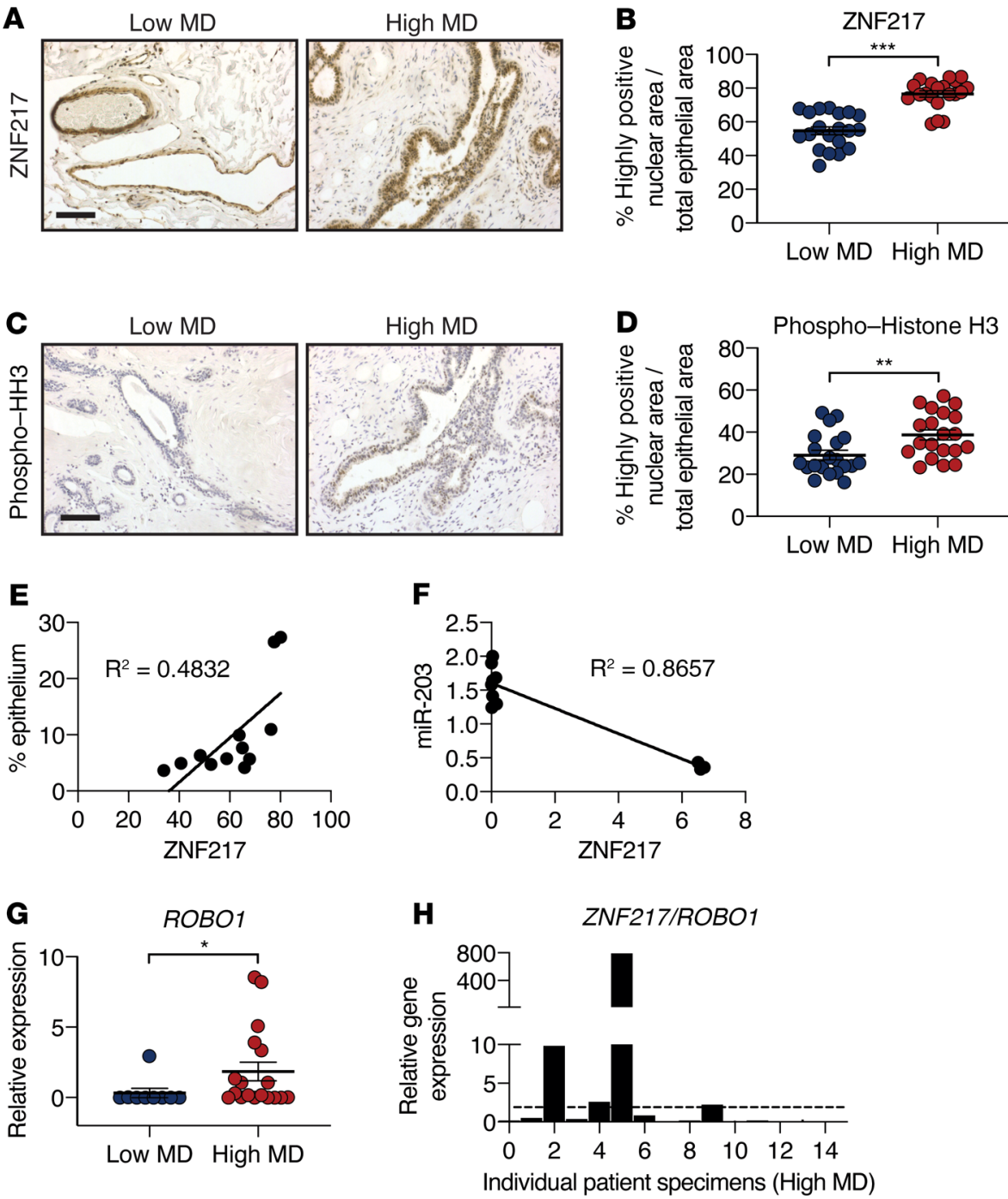

Figure 8. High ZNF217 expression and mammary epithelial cell proliferation are associated with high epithelial and mammographic density. (A) Immunohistochemical (IHC) staining of human breast tissue sections using a ZNF217-specific antibody. Scale bar: $100 \mu \mathrm{m}$. (B) Quantification of ZNF217 staining from A expressed as the percentage of highly positive epithelial nuclear area over total epithelial area (low MD, $n=20$; high MD, $n=20$ ). (C) IHC staining of human breast tissue sections using a phospho-histone H3-specific antibody. Scale bar: $100 \mu \mathrm{m}$. (D) Quantification of phospho-histone $\mathrm{H} 3$ staining from $\mathbf{C}$ expressed as the percentage of highly positive epithelial nuclear area over total epithelial area (low MD, $n=20$; high MD, $n=20)$. (E) Correlation between ZNF217 staining quantified in $\mathbf{B}$ and epithelial density $(n=12)$. (F) Correlation between ZNF217 and miR-203 levels determined by qRT-PCR from the same human breast specimens $(n=11)$. (C) qRT-PCR analysis for ROBO1 using RNA isolated from human breast tissues (low MD, $n=9$; high MD, $n=18$ ). Results are normalized to 185 RNA. (H) The ratio of relative $Z N F 217 / R O B 01$ gene expression for 14 individual patient specimens with high MD. The dashed line represents a ratio of 1 . Data are represented as mean \pm SEM. ${ }^{*} P<0.05$; ${ }^{* *} P<0.01$; ${ }^{* *} P<0.001$ by 2 -tailed Mann-Whitney $U$ test (B, D, and $\mathbf{G}$ ). increased expression of collagen cross-linking enzymes such as LOX and LH2, that could provide a tractable explanation for the thicker, bundled fibrillar collagens surrounding the periductal epithelium $(27,29,40,81)$. Importantly, quantitative mass spectrometry analysis of the insoluble ECM and associated matrisome distinguished all $4 \mathrm{MD}$ groups but could not detect any distinct qualitative differences between the groups, suggesting percentage stromal ECM and its posttranslational organization, as opposed to ECM specificity per se, likely distinguish these groups. Indeed, the prevalent morphological stromal traits associated with the higher-MD tissue included abundant fibrillar collagen, as detected by polarized imaging of picrosirius red-stained tissue; increased ECM orientation, as indicated by 2-photon and SIM-POL birefringence imaging; and increased collagen bundling and thickness, as revealed by SEM. Oriented, thicker, and more abundant fibrillar collagen contributes to ECM stiffness (30-32). Consistently, AFM indentation showed that the periductal breast stroma in the high-MD tissue was significantly stiffer than that measured in the low-MD tissue stroma. More importantly, analysis of high- versus low-MD tissue regions within the same normal, healthy human breast confirmed that a high-MD stroma is indeed significantly stiffer and contains a more oriented stromal ECM. The findings confirm prior studies in which a small cohort of high versus low $(n=3)$ tumor-associated breast tissue stroma was determined to be relatively stiffer and to contain thicker collagen bundles. Our studies extend these observations to include normal healthy tissue with the magnitude of the values showing better concordance with AFM measurements made by other investigators in normal and malignant human breast tissue (29). Accordingly, our results definitively establish that the stroma in the normal, healthy, highly mammographically dense breast contains more ECM proteins, including fibrillar collagens, that are organized into thicker and more oriented bundles and that confer greater mechanical properties and stiffness to the ECM.

We found that the mammary epithelium in the high-MD breast tissue expressed low levels of the tumor suppressor miR-203 and demonstrated how this could be mediated by the stiffer periductal stroma. The molecular mechanisms whereby a stiff ECM represses miR-203 remain unclear, but is likely mediated through pathways linked to integrin signaling and cytoskeletal tension such as $\beta$-catenin, myc, and TGF- $\beta(33,82)$. Indeed, we previously showed that a stiff ECM disproportionately increases the fraction of precursor miRs (33), and we recently determined that this effect can be ameliorated by reducing integrin signaling and actomyosin tension, 
suggesting cytoskeletal tension could influence the efficiency of miR processing (unpublished observations, Janna Mouw and Valerie Weaver). Alternately, ECM stiffness and cytoskeletal tension similarly regulate the levels and subcellular organization of several heterogeneous nuclear ribonucleoproteins (unpublished observations, Janna Mouw and Valerie Weaver) implicated in miR expression and processing $(83,84)$. Regardless of pathological origin, in the current study we showed that loss of miR-203 leads to a concomitant elevation of its target oncogene ZNF217 (60). We and others showed that ZNF217 activates PI3K/Akt to stimulate cell proliferation and motility and regulates the expression of TGF- $\beta$ and Wnt signaling pathway regulators that promote epithelial progenitor activity (refs. 57, 58, Figure 6, and Supplemental Figures 4 and 6). Not surprisingly, high ZNF217 levels promote breast tumor progression and metastasis in experimental mouse models of mammary cancer and its high expression in breast cancer associates with poor patient prognosis $(57,59,85)$. Our culture studies established a causal relationship between a highly fibrillar, stiff ECM BM/collagen gel, low miR-203, elevated MEC proliferation and high $Z N F 217 / Z f p 217$ expression and PI3K/Akt activity (Figure 6 and Supplemental Figures 4 and 6). Indeed, Zfp217 levels were also elevated in the proliferating, epithelia-dense, collagen-enriched mammary glands of a murine model of high MD that exhibits high tissue tensile strength, and epithelial proliferation could be reduced by inhibiting Akt activity (ref. 35 and Figure 7). Consistently, we observed that the elevated expression of ZNF217 in the human breast tissue with higher MD correlated positively with epithelial cell proliferation and high epithelial density and negatively with miR-203 levels (Figure 8). Although a stiff ECM may influence other proproliferative pathways or alter cell phenotypes through additional miR-203-targeted transcripts, these results identify ZNF217 as a potential mediator of the increased epithelial density associated with high-MD tissue and suggest that the stiffer stroma in these women induces qualitative changes in the epithelium that could contribute to their elevated lifetime risk of breast cancer.

Importantly and not surprisingly, given the large number of predicted miR targets, we determined that the stiffness-stimulated loss of miR-203 induced a concomitant increase in levels of one of its other validated targets, the tumor suppressor $\mathrm{ROBO1}$ (38). High levels of ROBO1, which inhibits cell motility and invasion, would oppose the ZNF217-induced malignant phenotype and help to maintain tissue homeostasis (38). In fact, the relative ratio of the expression levels of miR-203 targets, such as ZNF217 and $R O B O 1$, may ultimately govern the risk of malignancy in these high-MD breast tissues. Indeed, the tumor suppressor ROBO1 was deleted or methylated in 68 of 150 primary breast cancer cases $(45.3 \%)$ and mutated in a further $3 \%$ of breast cancers (cbioportal.org) (68-71). We observed a significantly elevated ZNF217/ $R O B O 1$ ratio in $24 \%$ of the high-MD human breast tissues examined, suggesting they may harbor MECs with methylated or mutated $R O B O 1$. Interestingly, the Akt inhibitor triciribine can inhibit the protumor, proliferative effect of ZNF217 and induces death in chemoresistant breast cancer cells (57). Therefore, triciribine or a similar compound could be a component of a new chemoprevention therapy to treat women with high MD that also have an abnormally elevated $Z N F 217 / R O B O 1$ ratio. Collectively, these findings illustrate how the mechanically primed high-MD breast stroma could alter both the nature and the abundance of the breast epithelium to increase breast cancer risk. We also identify ROBO1 as a key tumor suppressor whose reduced expression could identify in women with increased mammographically dense breast tissues those who are at highest risk for malignant transformation and are therefore ideal candidates for increased monitoring.

\section{Methods}

Human breast tissue and experimental design. Tissue specimens were collected from prophylactic mastectomy and either formalin-fixed and paraffin-embedded (FFPE) or flash frozen in OCT (Tissue-Tek), as described previously (30). Human patient samples were randomized and blinded for all analyses using deidentified patient B-numbers. All data, such as MD, were acquired without prior knowledge of patient information, which was only revealed following analysis.

Animals, animal care, and drug treatment. Animal husbandry for mice was carried out in Laboratory Animal Resource Center (LARC) facilities at UCSF. Mammary glands were harvested from euthanized 8to 10-week-old mice for the isolation of MECs for subsequent analysis. Mammary gland tissues from 10-week-old Col1a1 ${ }^{\text {tmIJae }}$ mice (63) were provided as FFPE blocks by the Department of Cell and Regenerative Biology, University of Wisconsin-Madison. Col1a1 ${ }^{\text {tmIJae }}$ mice (B6;129S4 background) were obtained from The Jackson Laboratory. Triciribine (APExBIO, catalog A8541) was diluted in DMSO and administered by intraperitoneal injection to 8 -week-old Col1a1 ${ }^{\text {tmIJae }}$ mice at a dosage of 1 $\mathrm{mg} / \mathrm{kg}, 5$ times per week, for a total of 2 weeks.

Proteomic sample preparation and analysis. See Supplemental Methods for details.

Immunohistochemistry. Immunohistochemical staining of FFPE human and mouse tissue specimens was performed as described previously (36). See Supplemental Methods for more details.

Immunofluorescence, image acquisition, and analysis. Immunofluorescence staining of human breast tissue specimens was performed as described previously $(34,37)$. See Supplemental Methods for more details.

Immunoblotting. Immunoblotting was performed as described previously (86) using the following antibodies: ZNF217 (MilliporeSigma, catalog HPA051857; 1:1000), phosphorylated Akt substrate $\left(\mathrm{RXXS}^{*} / \mathrm{T}^{*}\right)$ (110B7E) (Cell Signaling Technology, catalog 9614; 1:1000) and $\beta$-actin (MilliporeSigma, catalog A5441; 1:5000).

Picrosirius red and trichrome staining and quantification. Picrosirius red and trichrome staining of human breast tissues was performed as described previously $(30,87)$. Quantification of staining was done using specific Image (NIH) macros written to identify and quantify blue color for trichrome staining, or red in picrosirius-stained sections illuminated with polarized light to visualize fibrillar collagens.

Two-photon microscopy image acquisition and analysis. Two-photon microscopy, image rendering, and calculation of collagen fiber volumes and orientation was performed as previously described using Imaris (Bitplane AG) and MATLAB (MathWorks) (30, 82).

SIM-POL imaging and analysis. SIM-POL Imaging was performed as previously described $(30,88)$. Retardance maps were obtained using custom-written MATLAB programs to analyze tissue images acquire by an Olympus microscope (IX81) with $\times 10$ objective according to methods previously described (89).

$A F M$. AFM indentation maps were obtained as described previously $(30,90)$ using an MFP3D-BIO inverted optical atomic force 
microscope mounted on a Nikon TE2000-U inverted fluorescence microscope (Asylum Research). AFM maps were generated for a maximum of 40 minutes before tissues were fixed and stained for alternate analyses. Periductal ECM-rich regions were selected to generate all force maps and several maps were obtained for each patient specimen.

Cell culture, DNA constructs, and lentiviral transduction of cells. MCF10A cells (ATCC) were cultured as described previously (34) with the exception that acini in collagen gels were cultured with reduced levels of horse serum (2\%) and epidermal growth factor (EGF; $5 \mathrm{ng} /$ $\mathrm{mL}$ ). Lentiviral constructs for miR-203 manipulations were prepared as described previously $(37,91,92)$. Lentiviral shRNA constructs targeting ZNF217 have been previously described and were provided by the Harper Cancer Research Institute, University of Notre Dame (57). Lentivirus was produced using 239-T cells and viral packaging vectors as described previously (37). MCF10A cells were infected with virus-containing media and either selected with $1 \mu \mathrm{g} / \mathrm{mL}$ puromycin (for miR antagomir expression) or sorted by midrange or high levels of green fluorescent protein (GFP) with a BD FACSAria II cell sorter (for CTL and miR-203 ${ }^{\mathrm{OE}}$ vectors [lentiviral pLKO.1 puro vector, MilliporeSigma]) and ZNF217-shRNA vectors (57).

Mouse mammary organoid isolation. Mammary glands were harvested from 10- to 12-week-old WT FVB/n mice and chopped manually. Fragments were then transferred into 10 to $20 \mathrm{~mL}$ of Dulbecco's modified Eagle medium (DMEM, Gibco) supplemented with $2 \%$ fetal bovine serum (FBS), $2 \mathrm{mg} / \mathrm{mL}$ collagenase (MilliporeSigma), and $0.1 \mathrm{mg} / \mathrm{mL}$ hyaluronidase (MilliporeSigma) for a 1-hour incubation with shaking at $37^{\circ} \mathrm{C}$. Following digestion, fragments were subjected to a short (3 minute) digestion with $0.2 \%$ trypsin/EDTA. The resulting mammary organoids/ spheroids were washed with DMEM before their resuspension in BM/collagen hydrogels. Organoids were cultured in DMEM/F12 supplemented with $20 \mathrm{ng} / \mathrm{mL}$ EGF, $10 \mu \mathrm{g} / \mathrm{mL}$ insulin, and $2 \mu \mathrm{g} / \mathrm{mL}$ hydrocortisone.

$P A$ gels for cell culture. PA hydrogels were prepared as previously described $(93,94)$. Following functionalization with succinimidyl ester, hydrogels were conjugated overnight with $1 \mu \mathrm{g} / \mathrm{mL} \mathrm{BM}(\mathrm{BD})$ at $4^{\circ} \mathrm{C}$ and rinsed with PBS/DMEM before cell plating.

Collagen hydrogel preparation, cell culture assays, and RNA isolation. Collagen hydrogels were prepared from rat tail collagen I (Corning, catalog 354249). To cross-link and stiffen the BM/collagen, it was incubated for more than 10 days with $0.1 \%$ acetic acid containing $500 \mathrm{mM}$ L-ribose (STIFF) or $0.1 \%$ acetic acid alone (SOFT). BM/collagen was then mixed with $20 \%$ BM, DMEM, PBS and $1 \mu \mathrm{g} / \mathrm{mL}$ fibronectin. $1 \mathrm{~N} \mathrm{NaOH}$ was added to achieve a neutral $\mathrm{pH}$ and a thin base layer of $100 \mu \mathrm{L}$ volume was added to the well of a 48-well tissue culture plate. MCF10A or mouse mammary acini were resuspended in the SOFT and STIFF BM/collagen preparations and plated as a top layer of $100 \mu \mathrm{L}$ and allowed to solidify for 30 minutes at room temperature followed by 30 minutes at $37^{\circ} \mathrm{C}$. Cell medium was then added and gels were detached from the wells to float. Hydrogels and cells were resuspended in TRIzol/chloroform (Invitrogen) for subsequent RNA extraction using the Ambion mirVana kit (AM1560) per manufacturer's instructions.

qRT-PCR (miRs and $m R N A s$ ). Quantitative PCR (qPCR) analysis of miRs and mRNA was performed as previously described $(33,37)$. For mRNA analysis, $18 S$ primers were used to control for cDNA concentration in separate PCR reactions for each sample. LightCycler Fast Start DNA Master SYBR Green Mix (Roche) was added to each PCR reaction along with cDNA and 1 pmol primer in a total volume of $10 \mu \mathrm{L}$.
In situ hybridization. In situ hybridization was performed according to the manufacturer's protocol for miRCURY LNA miR Detection Probes and ISH Optimization Kit, as previously described (95).

SEM. Glass slides were cleaned using 0.1 M HCL, followed by 0.1 $\mathrm{M} \mathrm{NaOH}$ and washed with water. OCT sections $(20 \mu \mathrm{m})$ of breast tissue were cut and allowed to adhere to glass slides. Immediately before use, sections were warmed to room temperature and fixed and dehydrated as previously described $(96,97)$. Sections were dried using a critical-point dryer, followed by 8-nm sputter coating with either gold or gold/platinum before image acquisition on a Zeiss Ultra55 FE-SEM in the San Francisco State University Electron Microscopy Facility.

Statistics. Statistical calculations were performed using GraphPadPrism 8 software and assessed by 2-tailed unpaired Student's $t$ test, and 1-way or 2-way ANOVA (with Tukey's or Holm-Šidák's multiple-comparison correction) for grouped analyses after confirming that the data met appropriate assumptions. Nonparametric, 2-tailed Mann-Whitney $U$ tests or Kruskal-Wallis tests followed by Dunn's multiple-comparison test were used where appropriate. A $P$ value less than 0.05 was considered significant.

Study approval. All human breast tissue specimens were collected prospectively from informed and consenting patients undergoing surgery at UCSF or Duke University Medical Centers between 2010 and 2018. Samples were stored and analyzed with deidentified labels to protect patient data in accordance with the procedures outlined in the IRB protocol 10-03832, approved by the UCSF Committee of Human Resources and the Duke University IRB (Pro00034242). Animals were housed in the Laboratory Animal Resource Center (LARC) facilities at UCSF Parnassus in accordance with the guidelines stipulated by the Institutional Animal Care Use Committee (IACUC) protocols AN133001-03 and AN179766-01, which adhere to the NIH Guide for the Care and Use of Laboratory Animals (National Academies Press, 2011).

\section{Author contributions}

VMW conceived and designed the study. VMW, KCH, and JJN directed the studies. ASB executed the proteomics studies. IA and JJN conducted AFM analysis. IA and JJN conducted 2-photon and picrosirius red analysis. IA and QS conducted SIM-POL imaging under the supervision of JTL. ESH and RAM provided human breast tissue specimens. MKH performed H\&E and immunohistochemical staining and analysis of human breast tissues. YYC assessed tissue pathology. JNL prepared the lentiviral constructs for miR-203 manipulations. ISD, JKM, JJN, and ST conducted the miR analysis. JW and LEL stained and analyzed ZNF217 and p-HH3 levels in human tissues and provided lentiviral shRNA constructs targeting ZNF217. SMP and PJK provided the murine Col1a1 $1^{\text {tmae }}$ tissue. PJH quantified epithelial proliferation and density and ZNF217 and p-HH3 staining in human tissues. JAS conducted mammographic density imaging of prophylactic mastectomy tissue. VMW and JJN wrote the manuscript with editorial input from MKH, LEL, SS, and ASB.

\section{Acknowledgments}

We thank Lydia and Nataliya Korets for care and handling of animals. We also thank Nataliya Korets for tissue histology, the Nikon Imaging Center and Biological Imaging Development Center for microscopy support, as well as the Parnassus Flow Cytometry Core for support with cell sorting. This 
work was supported by US NIH/National Cancer Institute grants 1R01CA222508-01 (to VMW and ESH), R01CA192914 (to VMW), and R33CA206922 (to LEL), US Department of Defense (DOD) Breast Cancer Research Program (BCRP) grant BC122990 (to VMW), American Cancer Society Research Scholar Award RSG-17-206-01 (to LEL), Give Breast Cancer the Boot pilot project grant (to VMW and RAM), and American Association for Cancer Research (AACR) grants 15-40-01-NORT: Basic
Cancer Research Fellowship (to JJN), and 17-40-48-NORT: AACR Janssen Cancer Interception Research Fellowship (to JJN).

Address correspondence to: Valerie M. Weaver, Center for Bioengineering and Tissue Regeneration, Department of Surgery, University of California, San Francisco, 513 Parnassus, HSE 560, Box 0456, San Francisco, California 94143-0456, USA. Phone: 415.476.3826; Email: valerie.weaver@ucsf.edu.
1. Boyd NF, et al. Quantitative classification of mammographic densities and breast cancer risk: results from the Canadian National Breast Screening Study. J Natl Cancer Inst. 1995;87(9):670-675.

2. Boyd NF, Martin LJ, Yaffe MJ, Minkin S. Mammographic density and breast cancer risk: current understanding and future prospects. Breast Cancer Res. 2011;13(6):223.

3. Byrne C, et al. Mammographic features and breast cancer risk: effects with time, age, and menopause status. J Natl Cancer Inst. 1995;87(21):1622-1629.

4. McCormack VA, dos Santos Silva I. Breast density and parenchymal patterns as markers of breast cancer risk: a meta-analysis. Cancer Epidemiol Biomarkers Prev. 2006;15(6):1159-1169.

5. Palomares MR, Machia JR, Lehman CD, Daling JR, McTiernan A. Mammographic density correlation with Gail model breast cancer risk estimates and component risk factors. Cancer Epidemiol Biomarkers Prev. 2006;15(7):1324-1330.

6 . Vinnicombe SJ. Breast density: why all the fuss? Clin Radiol. 2018;73(4):334-357.

7. Li T, et al. The association of measured breast tissue characteristics with mammographic density and other risk factors for breast cancer. Cancer Epidemiol Biomarkers Prev. 2005;14(2):343-349.

8. Hawes D, et al. Dense breast stromal tissue shows greatly increased concentration of breast epithelium but no increase in its proliferative activity. Breast Cancer Res. 2006;8(2):R24.

9. Gabrielson M, et al. Amount of stroma is associated with mammographic density and stromal expression of oestrogen receptor in normal breast tissues. Breast Cancer Res Treat. 2016;158(2):253-261.

10. Ghosh K, et al. Tissue composition of mammographically dense and non-dense breast tissue. Breast Cancer Res Treat. 2012;131(1):267-275.

11. Bartow SA, Pathak DR, Mettler FA, Key CR, Pike MC. Breast mammographic pattern: a concatenation of confounding and breast cancer risk factors. Am J Epidemiol. 1995;142(8):813-819.

12. Boyd NF, Jensen HM, Cooke G, Han HL. Relationship between mammographic and histological risk factors for breast cancer. J Natl Cancer Inst. 1992;84(15):1170-1179.

13. Huo CW, et al. High mammographic density is associated with an increase in stromal collagen and immune cells within the mammary epithelium. Breast Cancer Res. 2015;17:79.

14. Turashvili G, et al. Columnar cell lesions, mammographic density and breast cancer risk. Breast Cancer Res Treat. 2009;115(3):561-571.

15. Boyd NF, Jensen HM, Cooke G, Han HL, Lockwood GA, Miller AB. Mammographic densities and the prevalence and incidence of histological types of benign breast disease. Reference Pathologists of the Canadian National Breast Screening Study. Eur J Cancer Prev. 2000;9(1):15-24.

16. Key TJ. Endogenous oestrogens and breast cancer risk in premenopausal and postmenopausal women. Steroids. 2011;76(8):812-815.

17. Byrne $C$, et al. Mammographic density change with estrogen and progestin therapy and breast cancer risk. J Natl Cancer Inst. 2017;109(9).

18. Cuzick J, et al. Tamoxifen-induced reduction in mammographic density and breast cancer risk reduction: a nested case-control study. J Natl Cancer Inst. 2011;103(9):744-752.

19. Li J, Humphreys K, Eriksson L, Edgren G, Czene K, Hall P. Mammographic density reduction is a prognostic marker of response to adjuvant tamoxifen therapy in postmenopausal patients with breast cancer. JClin Oncol. 2013;31(18):2249-2256.

20. Port ER, Montgomery LL, Heerdt AS, Borgen PI. Patient reluctance toward tamoxifen use for breast cancer primary prevention. Ann Surg Oncol. 2001;8(7):580-585.

21. Cuzick J, Sestak I, Thorat MA. Impact of preventive therapy on the risk of breast cancer among women with benign breast disease. Breast. 2015;24 suppl 2:S51-S55.

22. De Grève J, Sermijn E, De Brakeleer S, Ren Z, Teugels E. Hereditary breast cancer: from bench to bedside. Curr Opin Oncol. 2008;20(6):605-613.

23. Stone J, et al. The heritability of mammographically dense and nondense breast tissue. Cancer Epidemiol Biomarkers Prev. 2006;15(4):612-617.

24. Boyd NF, et al. Mammographic density: a heritable risk factor for breast cancer. Methods Mol Biol. 2009;472:343-360.

25. Dite GS, et al. Predictors of mammographic density: insights gained from a novel regression analysis of a twin study. Cancer Epidemiol Biomarkers Prev. 2008;17(12):3474-3481.

26. Lindström S, et al. Corrigendum: genome-wide association study identifies multiple loci associated with both mammographic density and breast cancer risk. Nat Commun. 2015;6:8358.

27. Alowami S, Troup S, Al-Haddad S, Kirkpatrick I, Watson PH. Mammographic density is related to stroma and stromal proteoglycan expression. Breast Cancer Res. 2003;5(5):R129-R135.

28. Boyd NF, et al. Evidence that breast tissue stiffness is associated with risk of breast cancer. PLoS One. 2014;9(7):e100937.

29. McConnell JC, et al. Increased peri-ductal collagen micro-organization may contribute to raised mammographic density. Breast Cancer Res. 2016;18(1):5

30. Acerbi I, et al. Human breast cancer invasion and aggression correlates with ECM stiffening and immune cell infiltration. Integr Biol (Camb).
2015;7(10):1120-1134.

31. Levental KR, et al. Matrix crosslinking forces tumor progression by enhancing integrin signaling. Cell. 2009;139(5):891-906.

32. Mouw JK, Ou G, Weaver VM. Extracellular matrix assembly: a multiscale deconstruction. Nat Rev Mol Cell Biol. 2014;15(12):771-785.

33. Mouw JK, et al. Tissue mechanics modulate microRNA-dependent PTEN expression to regulate malignant progression. Nat Med. 2014;20(4):360-367.

34. Paszek MJ, et al. Tensional homeostasis and the malignant phenotype. Cancer Cell. 2005;8(3):241-254.

35. Provenzano PP, et al. Collagen density promotes mammary tumor initiation and progression. BMC Med. 2008;6:11.

36. Barnes JM, et al. A tension-mediated glycocalyx-integrin feedback loop promotes mesenchymal-like glioblastoma. Nat Cell Biol. 2018;20(10):1203-1214.

37. Laklai H, et al. Genotype tunes pancreatic ductal adenocarcinoma tissue tension to induce matricellular fibrosis and tumor progression. Nat Med. 2016;22(5):497-505.

38. Le LT, et al. Loss of miR-203 regulates cell shape and matrix adhesion through ROBO1/Rac/FAK in response to stiffness. JCell Biol. 2016;212(6):707-719.

39. Northey JJ, Przybyla L, Weaver VM. Tissue force programs cell fate and tumor aggression. Cancer Discov. 2017;7(11):1224-1237.

40. Chiquet M, Birk DE, Bönnemann CG, Koch M. Collagen XII: Protecting bone and muscle integrity by organizing collagen fibrils. Int J Biochem Cell Biol. 2014;53:51-54.

41. Hicks D, et al. Mutations in the collagen XII gene define a new form of extracellular matrix-related myopathy. Hum Mol Genet. 2014;23(9):2353-2363.

42. Nissen NI, Karsdal M, Willumsen N. Collagens and cancer associated fibroblasts in the reactive stroma and its relation to cancer biology. J Exp Clin Cancer Res. 2019;38(1):115.

43. Sahai E, et al. A framework for advancing our understanding of cancer-associated fibroblasts. Nat Rev Cancer. 2020;20(3):174-186.

44. Gjaltema RA, Bank RA. Molecular insights into prolyl and lysyl hydroxylation of fibrillar collagens in health and disease. Crit Rev Biochem Mol Biol. 2017;52(1):74-95.

45. Maller O, et al. Inflammation promotes tumor aggression by stimulating stromal cell-dependent collagen crosslinking and stromal stiffening. Posted on bioRxiv February 13, 2020. https://doi. org/10.1101/2020.02.13.948141.

46. Greene SB, Herschkowitz JI, Rosen JM. Small players with big roles: microRNAs as targets to inhibit breast cancer progression. Curr Drug 
Targets. 2010;11(9):1059-1073.

47. Giordano L, Gallo F, Petracci E, Chiorino G, Segnan $\mathrm{N}$, Andromeda working group. The ANDROMEDA prospective cohort study: predictive value of combined criteria to tailor breast cancer screening and new opportunities from circulating markers: study protocol. BMC Cancer. 2017;17(1):785.

48. Shidfar A, et al. Expression of miR-18a and miR-210 in normal breast tissue as candidate biomarkers of breast cancer risk. Cancer Prev Res (Phila). 2017;10(1):89-97.

49. Goh JN, et al. microRNAs in breast cancer: regulatory roles governing the hallmarks of cancer. Biol Rev Camb Philos Soc. 2016;91(2):409-428.

50. He S, Zhang G, Dong H, Ma M, Sun Q. miR-203 facilitates tumor growth and metastasis by targeting fibroblast growth factor 2 in breast cancer. Onco Targets Ther. 2016;9:6203-6210.

51. Lin J, Wang L, Gao J, Zhu S. MiR-203 inhibits estrogen-induced viability, migration and invasion of estrogen receptor $\alpha$-positive breast cancer cells. Exp Ther Med. 2017;14(3):2702-2708.

52. Moes $\mathrm{M}$, et al. A novel network integrating a miRNA-203/SNAI1 feedback loop which regulates epithelial to mesenchymal transition. PLoS ONE. 2012;7(4):e35440.

53. Wang C, Zheng X, Shen C, Shi Y. MicroRNA-203 suppresses cell proliferation and migration by targeting BIRC5 and LASP1 in human triplenegative breast cancer cells. J Exp Clin Cancer Res. 2012;31:58.

54. Zhang Z, et al. Epigenetic silencing of miR-203 upregulates SNAI2 and contributes to the invasiveness of malignant breast cancer cells. Genes Cancer. 2011;2(8):782-791.

55. Dweep H, Gretz N. miRWalk2.0: a comprehensive atlas of microRNA-target interactions. Nat Methods. 2015;12(8):697.

56. Chou CH, et al. miRTarBase update 2018: a resource for experimentally validated microRNA-target interactions. Nucleic Acids Res. 2018;46(D1):D296-D302.

57. Littlepage LE, et al. The transcription factor ZNF217 is a prognostic biomarker and therapeutic target during breast cancer progression. Cancer Discov. 2012;2(7):638-651.

58. Nonet GH, Stampfer MR, Chin K, Gray JW, Collins CC, Yaswen P. The ZNF217 gene amplified in breast cancers promotes immortalization of human mammary epithelial cells. Cancer Res. 2001;61(4):1250-1254.

59. Plevova $P$, et al. CCND1 and ZNF217 gene amplification is equally frequent in BRCA1 and BRCA2 associated and non-BRCA breast cancer. Neoplasma. 2010;57(4):325-332.

60. Li Z, et al. MiR-203 suppresses ZNF217 upregulation in colorectal cancer and its oncogenicity. PLoS One. 2015;10(1):e0116170.

61. Huang G, et al. ZNF217 suppresses cell death associated with chemotherapy and telomere dysfunction. Hum Mol Genet. 2005;14(21):3219-3225.

62. Krig SR, et al. ZNF217, a candidate breast cancer oncogene amplified at 20q13, regulates expression of the ErbB3 receptor tyrosine kinase in breast cancer cells. Oncogene. 2010;29(40):5500-5510.

63. Liu X, Wu H, Byrne M, Jeffrey J, Krane S, Jaenisch R. A targeted mutation at the known collagenase cleavage site in mouse type I collagen impairs tis- sue remodeling. JCell Biol. 1995;130(1):227-237.

64. Shawky MS, Huo CW, Henderson MA, Redfern A, Britt K, Thompson EW. A review of the influence of mammographic density on breast cancer clinical and pathological phenotype. Breast Cancer Res Treat. 2019;177(2):251-276.

65. Nguyen TL, et al. Interval breast cancer risk associations with breast density, family history and breast tissue aging. Int $J$ Cancer . 2020;147(2):375-382.

66. Evans DGR, et al. Breast cancer pathology and stage are better predicted by risk stratification models that include mammographic density and common genetic variants. Breast Cancer Res Treat. 2019;176(1):141-148.

67. Miroshnikova YA, et al. Tissue mechanics promote IDH1-dependent HIF1 $\alpha$-tenascin C feedback to regulate glioblastoma aggression. Nat Cell Biol. 2016;18(12):1336-1345.

68. Bhattacharya R, et al. Frequent alterations of SLIT2-ROBO1-CDC42 signalling pathway in breast cancer: clinicopathological correlation. J Genet. 2016;95(3):551-563.

69. Cerami E, et al. The cBio cancer genomics portal: an open platform for exploring multidimensional cancer genomics data. Cancer Discov. 2012;2(5):401-404.

70. Dallol A, et al. Tumour specific promoter region methylation of the human homologue of the Drosophila Roundabout gene DUTT1 (ROBO1) in human cancers. Oncogene. 2002;21(19):3020-3028.

71. Gao J, et al. Integrative analysis of complex cancer genomics and clinical profiles using the cBioPortal. Sci Signal. 2013;6(269):pl1.

72. Boyd N, et al. The origins of breast cancer associated with mammographic density: a testable biological hypothesis. Breast Cancer Res. 2018;20(1):17.

73. Harvey JA, et al. Histologic changes in the breast with menopausal hormone therapy use: correlation with breast density, estrogen receptor, progesterone receptor, and proliferation indices. Menopause. 2008;15(1):67-73.

74. Khan QJ, Kimler BF, O’Dea AP, Zalles CM, Sharma P, Fabian CJ. Mammographic density does not correlate with Ki-67 expression or cytomorphology in benign breast cells obtained by random periareolar fine needle aspiration from women at high risk for breast cancer. Breast Cancer Res. 2007;9(3):R35.

75. Pang JM, et al. Breast tissue composition and immunophenotype and its relationship with mammographic density in women at high risk of breast cancer. PLoS One. 2015;10(6):e0128861.

76. Verheus M, et al. Mammographic density and epithelial histopathologic markers. BMC Cancer. 2009;9:182.

77. Guo YP, et al. Growth factors and stromal matrix proteins associated with mammographic densities. Cancer Epidemiol Biomarkers Prev. 2001;10(3):243-248.

78. Barrett AS, et al. Hydroxylamine chemical digestion for insoluble extracellular matrix characterization. J Proteome Res. 2017;16(11):4177-4184.

79. Goddard ET, et al. Quantitative extracellular matrix proteomics to study mammary and liver tissue microenvironments. Int J Biochem Cell Biol. 2016;81(pt A):223-232.

80. Hill RC, Calle EA, Dzieciatkowska M, Niklason LE, Hansen KC. Quantification of extracellular matrix proteins from a rat lung scaffold to provide a molecular readout for tissue engineering. Mol Cell Proteomics. 2015;14(4):961-973.

81. Flück M, Giraud MN, Tunç V, Chiquet M. Tensile stress-dependent collagen XII and fibronectin production by fibroblasts requires separate pathways. Biochim Biophys Acta. 2003;1593(2-3):239-248.

82. Pickup MW, et al. Stromally derived lysyl oxidase promotes metastasis of transforming growth factor- $\beta$-deficient mouse mammary carcinomas. Cancer Res. 2013;73(17):5336-5346.

83. Geuens T, Bouhy D, Timmerman V. The hnRNP family: insights into their role in health and disease. Hum Genet. 2016;135(8):851-867.

84. Jean-Philippe J, Paz S, Caputi M. hnRNP A1: the Swiss army knife of gene expression. Int J Mol Sci. 2013;14(9):18999-19024.

85. Bai WD, et al. MiR-200c suppresses TGF- $\beta$ signaling and counteracts trastuzumab resistance and metastasis by targeting ZNF217 and ZEB1 in breast cancer. Int JCancer. 2014;135(6):1356-1368.

86. Johnson KR, Leight JL, Weaver VM. Demystifying the effects of a three-dimensional microenvironment in tissue morphogenesis. Methods Cell Biol. 2007;83:547-583.

87. Király K, et al. Specimen preparation and quantification of collagen birefringence in unstained sections of articular cartilage using image analysis and polarizing light microscopy. Histochem J. 1997;29(4):317-327.

88. Shin IH, Shin SM, Kim DY. New, simple theorybased, accurate polarization microscope for birefringence imaging of biological cells. J Biomed Opt. 2010;15(1):016028.

89. Glazer AM, Lewis JG, Kaminsky W. An automatic optical imaging system for birefringent media. Proc R Soc Lond A. 1996;452(1955):2751-2765.

90. Lopez JI, Kang I, You WK, McDonald DM, Weaver VM. In situ force mapping of mammary gland transformation. Integr Biol (Camb). 2011;3(9):910-921.

91. Scherr M, et al. Lentivirus-mediated antagomir expression for specific inhibition of miRNA function. Nucleic Acids Res. 2007;35(22):e149.

92. Stegmeier F, Hu G, Rickles RJ, Hannon GJ, Elledge SJ. A lentiviral microRNA-based system for single-copy polymerase II-regulated RNA interference in mammalian cells. Proc Natl Acad Sci U S A. 2005;102(37):13212-13217.

93. Lakins JN, Chin AR, Weaver VM. Exploring the link between human embryonic stem cell organization and fate using tension-calibrated extracellular matrix functionalized polyacrylamide gels. Methods Mol Biol. 2012;916:317-350.

94. Przybyla L, Lakins JN, Sunyer R, Trepat X, Weaver VM. Monitoring developmental force distributions in reconstituted embryonic epithelia. Methods. 2016;94:101-113.

95. Jørgensen S, Baker A, Møller S, Nielsen BS. Robust one-day in situ hybridization protocol for detection of microRNAs in paraffin samples using LNA probes. Methods. 2010;52(4):375-381.

96. Albuschies J, Vogel V. The role of filopodia in the recognition of nanotopographies. Sci Rep. 2013;3:1658.

97. Bos FL, Hawkins JS, Zovein AC. Single-cell resolution of morphological changes in hemogenic endothelium. Development. 2015;142(15):2719-2724. 